\title{
Efficient Certificateless Anonymous Multi-Receiver Encryption Scheme without Bilinear Parings
}

\author{
Ronghai Gao $\mathbb{D},{ }^{1}$ Jiwen Zeng $\mathbb{D},{ }^{1}$ and Lunzhi Deng $\mathbb{D}^{2}$ \\ ${ }^{1}$ School of Mathematical Sciences, Xiamen University, Xiamen 361005, China \\ ${ }^{2}$ School of Mathematical Sciences, Guizhou Normal University, Guiyang 550001, China \\ Correspondence should be addressed to Ronghai Gao; gaorh1978@163.com
}

Received 9 May 2018; Accepted 11 July 2018; Published 24 July 2018

Academic Editor: Giuseppe D’Aniello

Copyright (c) 2018 Ronghai Gao et al. This is an open access article distributed under the Creative Commons Attribution License, which permits unrestricted use, distribution, and reproduction in any medium, provided the original work is properly cited.

\begin{abstract}
With the growing development of Internet technology and popularization of mobile devices, we easily access the Internet anytime and anywhere by mobile devices. It has brought great convenience for our lives. But it brought more challenges than traditional wired communication, such as confidentiality and privacy. In order to improve security and privacy protection in using mobile network, numerous multi-receiver identity-based encryption schemes have been proposed with bilinear pairing and probabilistic hap-to-point (HTP) function. To address the troubles of private key escrow in multi-receiver encryption scheme based on ID-PKC, recently, some certificateless anonymous multi-receiver encryption (CLAMRE) schemes are introduced. But previous CLAMRE schemes using the bilinear pairing are not suitable to mobile device because the use of bilinear pairing and probabilistic hashto-point (HTP) function results in expensive operation costs in encryption or decryption. In this paper, we propose an efficient CLAMRE scheme using elliptic curve cryptography (ECC) without bilinear pairing and HTP hash function. Since our scheme does not use bilinear pairing and HTP operation during the encryption and decryption process, the proposed CLAMRE scheme has much less computation cost than the latest CLAMRE schemes. Performance analysis shows that runtime of our scheme is much less when the sender generates ciphertext, compared with existing schemes. Security analysis shows proposed CLAMRE scheme provides confidentiality of message and receiver anonymity under the random oracle model with the difficulties of decision DiffieHellman problem and against the adversaries defined in CL-PKC system.
\end{abstract}

\section{Introduction}

With the rapid development of the Internet technology and wireless communications and the popularity of mobile devices, we can access the Internet freely anytime and anywhere using mobile devices. This brings great convenience to our lives by Internet services. But we have to face the security problems of the openness of the wireless network. How to protect the security and privacy of wireless communications using mobile devices has been extensively considered by scholars. In order to achieve this goal, many encryption schemes (Fu Z et al. [1, 2]; Xia Z et al. [3]; Huang X et al. [4]), authentication schemes (Guo P et al. [5]; Shen J et al. [6]; Huang X et al. [4, 7];) and signature schemes (Ren $\mathrm{Y}$ et al. [8]; Wang J et al. [9]; Lee C C et al. [10]) have been proposed in recent years.
The multi-receiver encryption (MRE) or broadcast encryption (BEN) scheme is an important cryptographic primitive, in which a sender produces an identical ciphertext $\sigma$ by enciphering message $m$ and then sends $\sigma$ to group $S$ of selected receivers, and anyone in the group $S$ can decrypt the received ciphertext using his/her private key, and any user outside the privileged set $\mathrm{S}$ should not be able to recover the message. In fact, the application of multi-receiver confidential communication is very extensive, such as pay TV, video on demand, software protect, distribution of copyrighted material, and online gaming. When transmitting encrypted information to a public channel, the confidentiality of the information and the anonymity of the receiver are greatly challenged. The confidentiality is that only the authorized receiver can decrypt ciphertext $\sigma$ and message $m$ correctly. On the other hand, identity protection means that any 
receiver of the group cannot identify the identity of other receivers. MRE scheme is suitable for protecting the users' security and privacy. Therefore, it is necessary to consider how to design efficient and secure broadcast encryption and multiple receivers encryption scheme. In order to meet security requirements of the practical application, many MRE schemes (Kurosawa K [11]; Bellare M et al. [12]; Dodis Y et al. [13]; Kurosawa K [14]; Bellare M [15]) were proposed using the public key infrastructure (PKI).

In multi-receivers encryption schemes [11-15], existing management, distribution, and revocation of public key certificate need to bear huge storage space and high computing cost. To solve this problem, Beak et al. [16] constructed an efficient multi-receivers identity-based encryption (ID-based MRE); only one bilinear pair is required to encrypt a single message for $n$ receivers. In 2006, Chatterjee $S$ et al. [17] proposed a multi-receiver identity-based key encapsulation mechanism with security in the full model and sublinear size ciphertext. In this scheme, a controllable trade-off is achieved between the ciphertext size and the private size. However, Park et al. gave a way to attack the scheme of Chatterjee $S$ [17] and proved that it is not secure. In 2006, another IBBE scheme is designed by Yang et al. [18] using elliptic curve bilinear paring. However, they did not consider joining and departure of the recipient's membership in the design process, so the scheme was not suitable for a dynamic set. In scheme [16-18], the application scenario is single domain environment; that is, $n$ receivers come from the same management domain. However, in realistic applications, usually $n$ receivers will come from different management domains and they need once the bilinear pairing computation for one message, so their scheme becomes inefficient. In 2014, Wang $\mathrm{H}$ et al. [19] proposed an efficient multiple domain multireceiver identity-based encryption scheme that only requires one pairing computation to encrypt a single message for $n$ receivers from different administrative domains.

However, the above ID-based MRE schemes [16-19] cannot consider the receiver anonymity. To achieve preserving privacy of receivers, in 2010, Fan et al. [20] presented a new ID-based MRE scheme and claimed that it can protect receiver anonymity; the scheme is highly efficient for each receiver as it requires only two pairing operations. In 2012, Chien [21] found that the scheme of Fan et al. [20] failed to protect receiver anonymity and proposed an improved scheme which proves that the scheme enhances security and protects the anonymity of recipients. It is very unfortunate that Wang [22] pointed out the fact that Chien's scheme does not satisfy the indiscernibility of encryption under selective multi-identity, chosen ciphertext attacks. In 2015, Zhang [23] proposed the most efficient anonymous MRIBE scheme in terms of computational cost and communication overhead, compared with schemes of [20-22].

Although the above ID-based MRE schemes have many advantages, all of them face the problem of the private key escrow, which means that key generator center (KGC) calculates private key for every user by user identity and master private key of KGC; KGC retains all users private key; thus the user's privacy is easy to be leaked if KGC is not fully trusted. In order to address this security weakness, in 2003, Al-Riyami et al. [24] introduced the concept of the certificateless cryptography (CLC). In the CLC, the users' private key contains two parts: KGC and the user generate a partial private key and a secret value, respectively. Based on Al-Riyami et al's work, most certificateless signature (encryption) schemes [25-29] are proposed. In the existing research literature, certificateless multi-receiver encryption (CLMRE) scheme did not get more attention; Islam et al. [27] presented the concept of certificateless anonymous multi-receiver encryption (CLAMRE) and proposed the first CLAMRE scheme using the elliptic curve cryptography (ECC). Hung et al. [28] pointed out that scheme of [27] is less efficient and is not suitable in mobile devices environment, because the cost of encryption calculation is square of number of recipients, and proposed a new CLAMRE using the bilinear pairing. However, Hung et al.s CLAMRE scheme still does not suit mobile devices because of using bilinear pairing. In encryption, the sender that needs to operate bilinear pairs grows linearly because of the increase in the receivers' number.

Our Contribution. In this paper, we propose an efficient CLAMRE scheme using elliptic curve cryptography (ECC) without bilinear pairing and MTP hash function. Since our scheme does not use bilinear pairing and HTP operation during the encryption process, the proposed CLAMRE scheme has much less computation cost than the latest CLAMRE schemes; runtime of our scheme is much less in both encryption and decryption, compared with existing scheme $[28,29]$. Our scheme provides confidentiality of message and anonymity of receiver under the random oracle model with the difficulties of computational Diffie-Hellman problem and against the adversaries defined in CL-PKC system.

In this paper, we propose an efficient CLAMRE scheme using elliptic curve cryptography (ECC) without bilinear pairing and MTP hash function. Since our scheme does not use bilinear pairing and HTP operation during the encryption process, the proposed CLAMRE scheme has much less computation cost than the latest CLAMRE schemes; runtime of our scheme is much less in both encryption and decryption, compared with existing scheme $[28,29]$. Our scheme provides confidentiality of message and anonymity of receiver under the random oracle model with the difficulties of computational Diffie-Hellman problem and against the adversaries defined in CL-PKC system.

Organization. The rest of the paper is organized as follows. Mathematical preliminaries are introduced in Section 2. Formal definition of our CLAMRE scheme is presented in Section 3. Our CLAMRE scheme is proposed in Section 4. In Section 5, we give some security analysis of our CLAMRE scheme. Some performance analysis of our CLAMRE scheme is given in Section 6. At last, some conclusions of the paper are presented.

The rest of the paper is organized as follows. Mathematical preliminaries are introduced in Section 2. Formal definition of our CLAMRE scheme is presented in Section 3. Our CLAMRE scheme is proposed in Section 4. In Section 5, we give some security analysis of our CLAMRE scheme. Some performance analysis of our CLAMRE scheme is given 
in Section 6. At last, some conclusions of the paper are presented.

\section{Mathematical Preliminaries}

Here, we introduced the basic theory about the elliptic curve and existing some intractable problems.

2.1. Elliptic Curve. Suppose that $F_{p}$ is a finite field determined by a prime number $p$. The elliptic curve $E\left(F_{p}\right)$ over $F_{p}$ is the set of solutions $(x, y) \in F_{p} \times F_{p}$ to the congruence $y^{2} \equiv x^{3}+$ $a x+b(\bmod p)$, where $a, b \in F_{p}$ are constants such that $4 a^{3}+$ $27 b^{2} \neq 0(\bmod p)$, together with a special point $\mathcal{O}$ called the point at infinity or zero point.

The addition operation "+" on $E$ is defined as follows (where all arithmetic operations are performed in $F_{p}$ ): the point at infinity, $\mathcal{O}$, will be the identity element, so $P+\mathcal{O}=$ $\mathcal{O}+P=P$ for arbitrary $P \in E$.

Suppose $P, Q \in E$, if $P \neq Q$ and reflection of the point $P$ with respect to the $x$-axis is not the point $Q$; let $l$ be the line through $P$ and $Q$; otherwise $P=Q$; we define $l$ to be the tangent line through the point $P$. We denote $R^{\prime}$ as the third point in which $l$ intersects $E$; if we reflect $R^{\prime}$ in the $x$-axis, then we get a point which we call $R$. We define the following: $P+Q=R$. If reflection of the point $P$ with respect to the $x$ axis is point $Q$, let $Q=-P$; we define the following: $P+Q=$ $P+(-P)=P-P=\mathcal{O}$. The scalar point multiplication of the elliptic curve $E$ is defined as $t P=P+P+\cdots+P(t$ times $)$. Point $P$ has order $n$ if $n$ is the smallest positive integer such that $n P=\mathcal{O}$. So $(E,+)$ is an abelian group.

2.2. Computational Problems and Some Assumptions. Here, we mainly introduce the definitions of negligible function, decision Diffie-Hellman problem, and discrete logarithm (DL) problem, and assumptions are given.

Negligible Function. We call function $\omega(k)$ negligible if, for every $a>0$, there exists $l_{0}$ such that $\omega(k) \leq 1 / k^{l}$ for every $l \geq l_{0}$.

We call function $\omega(k)$ negligible if, for every $a>0$, there exists $l_{0}$ such that $\omega(k) \leq 1 / k^{l}$ for every $l \geq l_{0}$.

Discrete Logarithm (DL) Problem. Given a random instance $(P, x P)$, where $P \in E$, and $x \in Z_{p}^{*}$, computation of $x$ is computationally hard by a polynomial time-bounded algorithm. The probability that a polynomial time-bounded algorithm $\mathscr{A}$ can solve the DL problem is defined as $A d v_{\mathscr{A}}^{D L}(k)=$ $\operatorname{Pr}\left[\mathscr{A}(P, x P)=x: P \in E ; x \in Z_{p}^{*}\right]$.

Given a random instance $(P, x P)$, where $P \in E$, and $x \in Z_{p}^{*}$, computation of $x$ is computationally hard by a polynomial time-bounded algorithm. The probability that a polynomial time-bounded algorithm $\mathscr{A}$ can solve the DL problem is defined as $A d v_{\mathscr{A}}^{D L}(k)=\operatorname{Pr}[\mathscr{A}(P, x P)=x: P \in$ $\left.E ; x \in Z_{p}^{*}\right]$.

Discrete Logarithm (DL) Assumption. For any probabilistic polynomial time-bounded algorithm $\mathscr{A}, A d v_{\mathscr{A}}^{D L}(k)$ is negligible if $A d v_{\mathscr{A}}^{D L}(k) \leq \omega$, for negligible function $\omega$.
For any probabilistic polynomial time-bounded algorithm $\mathscr{A}, A d v_{\mathscr{A}}^{D L}(k)$ is negligible if $A d v_{\mathscr{A}}^{D L}(k) \leq \omega$, for negligible function $\omega$.

Decision Diffie-Hellman (DDH) Problem. Suppose that $P$ is point with order $p$ on $E$, and $A=a P, B=b P, X$ are random points on $\langle P\rangle$, where $a, b \in Z_{p}^{*}$. Determining if $X=$ $a b P$ holds is hard by a polynomial time-bounded algorithm. The probability that a polynomial time-bounded algorithm $\mathscr{A}$ can solve the DDH problem is defined as $A d v_{\mathscr{A}}^{D D H}(k)=$ $|\operatorname{Pr}[\mathscr{A}(P, a P, b P, X) \mid X=a b P]-1 / 2|$

Suppose that $P$ is point with order $p$ on $E$, and $A=$ $a P, B=b P, X$ are random points on $\langle P\rangle$, where $a, b \in Z_{p}^{*}$. Determining if $X=a b P$ holds is hard by a polynomial timebounded algorithm. The probability that a polynomial timebounded algorithm $\mathscr{A}$ can solve the DDH problem is defined as $A d v_{\mathscr{A}}^{D D H}(k)=|\operatorname{Pr}[\mathscr{A}(P, a P, b P, X) \mid X=a b P]-1 / 2|$

Decision Diffie-Hellman Assumption. For any probabilistic polynomial time-bounded algorithm $\mathscr{A}, A d v_{\mathscr{A}}^{D D H}(k)$ is negligible if $A d v_{\mathscr{A}}^{D D H}(k) \leq \omega$, for negligible function $\omega$.

For any probabilistic polynomial time-bounded algorithm $\mathscr{A}, A d v_{\mathscr{A}}^{C D H}(k)$ is negligible if $A d v_{\mathscr{A}}^{D D H}(k) \leq \omega$, for negligible function $\omega$.

\section{Formal Definition of the CLAMRE Scheme}

The CLAMRE scheme includes three categories of participants, that is, the sender of information, the private key generation center, and the group of selective receivers, respectively.

We denote $T=\left\{\mathscr{R}_{1}, \mathscr{R}_{2}, \cdots, \mathscr{R}_{n}\right\}$ as group of $n$ receivers selected by sender, $I D=\left\{I D_{1}, I D_{2}, \cdots, I D_{n}\right\}$ are their group identities, $p k=\left\{p k_{1}, p k_{2}, \cdots, p k_{n}\right\}$ are group public key, and $s k_{1}, s k_{2}, \cdots, s k_{n}$ are the full private key. In CLAMRE scheme, sender generates ciphertext $\sigma$ for message $m$ using public key $\left\{p k_{1}, p k_{2} \cdots, p k_{n}\right\}$ and identities $\left\{I D_{1}, I D_{2}, \cdots, I D_{n}\right\}$ of receivers $\left\{\mathscr{R}_{1}, \mathscr{R}_{2}, \cdots, \mathscr{R}_{n}\right\}$. Ciphertext $\sigma$ is conveyed to the receiver through the public channel. Every receiver $\mathscr{R}_{i}$ in group $T$ can correctly decrypt ciphertext $\sigma$ by using private key $s k_{i}$ for $i \in\{1,2, \cdots, n\}$. And arbitrary two receivers $R_{i}, R_{j}(i \neq j)$ in selected receiver group $T$ do not disclose the identity with each other. Figure 1 demonstrates intuitively the process of CLAMRE scheme. In the following, we depict the definition of the CLAMRE scheme.

In generally, a certificateless anonymous multi-receiver encryption scheme consists of a tuple (Setup, Partial Private-Key-Extract, Set-Secret - Value, Set - PrivateKey, Set - Public - Key, Multi - encryption, andMulti decryption.)

(i) Setup: selecting a security parameter $k$ as input, semitrusted private key generation center (KGC) executes this algorithm to generate the system's public parameters $\Omega$ and KGC's the master public/private key pair ( $m p k, m s k)$. $\Omega$ andmpk are published, and the master private $m s k$ is kept by KGC.

(ii) Partial - Private - Key - Extract: this algorithm is executed by KGC, according to the identity $I D_{i}$ of 


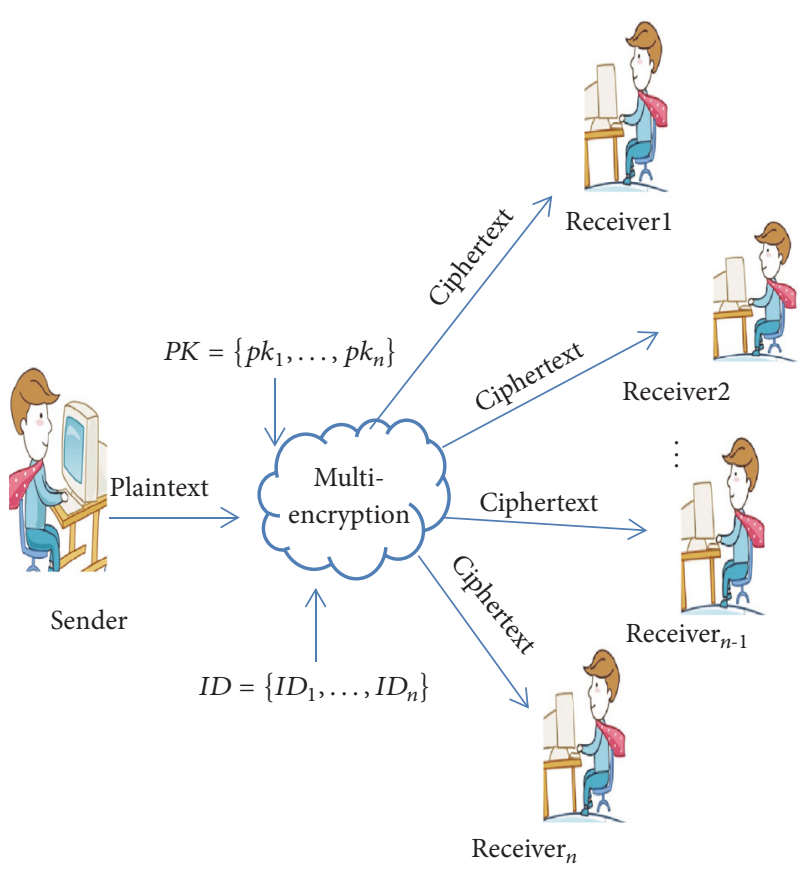

Figure 1: Process of a CLAMRE scheme.

receiver $\mathscr{R}_{i}$; the PKG computes the corresponding partial private key $s_{I D_{i}}$ using the master private key and delivers it to receiver $\mathscr{R}_{i}$ via an secure channel.

(iii) Set - secret - value: this algorithm is executed by receiver with identity $I D_{i}$ himself/herself to generate his/her secret value $t_{i}$.

(iv) Set - Private - Key: this algorithm is executed by receiver $\mathscr{R}_{i}$ with identity $I D_{i}$. It takes $\left(\Omega, s_{I D_{i}}, t_{i}\right)$ as input and returns the full private key $s k_{i}$ to $\mathscr{R}_{i}$ as output.

(v) Set - Public - Key: this algorithm is executed by receiver $\mathscr{R}_{i}$ himself/herself to generate his/her public key $p k_{i}$ according to his/her secret value $t_{i}$.

(vi) Multi - encryption:this is PPT algorithm. Sender executes this algorithm to generate a ciphertext for message $m$ by identities and full public of selected receivers.

(vii) Multi - decryption: a selected receiver runs this algorithm to decrypt the received ciphertext using the receivers full private key.

\section{Description of the Proposed CLAMRE Scheme}

In this section, we introduced our certificateless anonymous multi-receiver encryption (CLAMRE) scheme using elliptic curve cryptography (ECC) without bilinear pairings. The proposed scheme has three kinds of participants, i.e., a sender $S$, set $T$ consisting of selected $n$ receivers $\mathscr{R}_{1}, \mathscr{R}_{2}, \cdots, \mathscr{R}_{n}$, and a KGC. Sender generates ciphertext $\sigma$ by encrypting message $m$ only for selected receivers $\mathscr{R}_{1}, \mathscr{R}_{2}, \cdots, \mathscr{R}_{n}$; then sender delivers the ciphertext $\sigma$ to the receivers. Every receiver $R_{i}$ in $T$ can correctly decrypt ciphertext $\sigma$ receive by using his/her full private key $s k_{i}$ for $i \in\{1,2, \cdots, n\}$. And arbitrary two receivers $R_{i}, R_{j}(i \neq j)$ in selective receiver set $T$ do not disclose the identity with each other. The PKG generates the systems parameter and identity-based partial private keys of all the receivers $R_{i}$ for $i \in\{1,2, \cdots, n\}$. The proposed scheme includes the following seven algorithms (Setup, Partial - Private - Key - Extract, Set - Secret Value, Set - Private - Key, Set - Public - Key, Multi encryption, andMulti-decryption.)

(i) Setup: With the given security parameter $k$, this algorithm is executed KGC to generate the system's parameters. The following steps will be implemented $\mathrm{KGC}$ in this algorithm.

(1) Choose two $k$-bits prime integers $p$, $q$, two $k$-bits integers $l_{1}, l_{2}$, and an elliptic curve $E$ defined on $F_{p}$. Let $G$ be additive group on elliptic curve $E$, and $G_{q}$ be subgroup of $G$ with prime order $q$.

(2) Select randomly a generator $P \in G_{q}$.

(3) Randomly choose $x \in_{R} Z_{q}^{*}$ as the master key and $P_{\text {pub }}=x \cdot P$.

(4) Select four secure one-way hash functions $H_{i}$ : $\{0,1\}^{*} \longrightarrow Z_{q}^{*}(i=1,2,3) ; H_{4}:\{0,1\}^{*} \longrightarrow$ $\{0,1\}^{l_{1}+l_{2}}$.

(5) Publish system's parameters $\Omega=\left\{p, q, l_{1}, l_{2}, E\right.$, $\left.G, G_{q}, P, P_{p u b}, H_{1}, H_{2}, H_{3}, H_{4}\right\}$ and message space $M=\{0,1\}^{l_{1}}$.

(ii) Set-secret-value: A receiver $\mathscr{R}_{i}$ with $I D_{i}$ randomly selects $t_{i} \in Z_{q}^{*}$ as his or her secret value and computes $P K_{I D_{i}}=t_{i} \cdot P$ as the corresponding public key, and $\mathscr{R}_{i}$ sends $\left(P K_{I D_{i}}, I D_{i}\right)$ to KGC.

(iii) Partial - Private - Key - Extract: According to the identity $I D_{i}$ of receiver $\mathscr{R}_{i}$, the KGC performs the following steps:

(1) Randomly choose $r_{i} \in_{R} Z_{q}^{*}$ and compute $R_{I D_{i}}=$ $r_{i} \cdot P$.

(2) Calculate $k_{I D_{i}}=H_{1}\left(R_{I D_{i}}, P K_{I D_{i}}, I D_{i}\right)$ and $s_{I D_{i}}=$ $r_{i}+k_{I D_{i}} x \bmod (q)$

(3) The tuple $\left(R_{I D_{i}}, s_{I D_{i}}\right)$ is delivered to receiver $R_{i}$ by authenticated secure channel.

Here, $s_{I D_{i}}$ is receiver $\mathscr{R}_{i}$ 's partial private key. Partial private key $s_{I D}$ is valid if verify that equation $s_{I D_{i}} P=R_{I D_{i}}+H_{1}\left(R_{I D_{i}}, P K_{I D_{i}}, I D_{i}\right) P_{\text {pub }}$ is true and vice versa. Since we have

$$
\begin{gathered}
R_{I D_{i}}+H_{1}\left(R_{I D_{i}}, P K_{I D_{i}}, I D_{i}\right) P_{p u b}=r_{i} P+k_{I D_{i}} P_{p u b} \\
=r_{i} P+k_{I D_{i}} x P=\left(r_{i}+k_{I D_{i}} x\right) P=s_{I D_{i}} P
\end{gathered}
$$

(iv) Set - Private - Key: Receiver $\mathscr{R}_{i}$ secret keeps $s k_{i}=$ $\left(d_{i}, t_{i}\right)$ as his or her the full private.

(v) Set - Public - Key: Reciever $\mathscr{R}_{i}$ keeps $p k_{i}=\left(R_{I D_{i}}\right.$, $\left.P K_{I D_{i}}\right)$ as full public key. 
(vi) Multi - encryption: This algorithm is executed by sender $\delta$ to generate a ciphertext for given message $m$ and selected $n$ receivers $\mathscr{R}_{1}, \mathscr{R}_{2}, \cdots, \mathscr{R}_{n}$ with identity $I D_{1}, I D_{2}, \cdots I D_{n}$ respectively. The following steps will be performed in this algorithm.

(1) Choose randomly $\omega \in\{0,1\}^{l_{2}}$ and given message $m \in M$. Calculate $s=H_{2}(m, \omega)$ and $S=s P$.

(2) Compute $U_{i}=s \cdot\left(R_{I D_{i}}+k_{I D_{i}} P_{p u b}+P K_{I D_{i}}\right)$ and $\mu_{i}=H_{3}\left(U_{i}, I D_{i}, p k_{i}\right)$, where $i=1,2, \cdots, n$.

(3) Randomly select $e \epsilon_{R} Z_{q}^{*}$ and compute a polynomial $f(x)$ with degree $n$ as follows:

$$
\begin{aligned}
f(x) & =\prod_{i=1}^{n}\left(x-\mu_{i}\right)+e(\bmod q) \\
& =x_{n}+a_{n-1} x^{n-1}+\cdots+a_{1} x+a_{0},
\end{aligned}
$$

$$
\text { where } a_{i} \in Z_{q}^{*}(i=0,1, \ldots, n-1)
$$

(4) Compute $C=H_{4}(S, e) \oplus(m \| \omega)$

(5) Generate ciphertext $\sigma=(S, C, f)$.

(vii) Multi - decryption: This algorithm is executed by selected receiver $\mathscr{R}_{i}$ to extract plaintext from the received ciphertext $\sigma=(S, C, f) . \mathscr{R}_{i}$ performs following steps:

(1) Compute $U_{i}=\left(s_{I D_{i}}+t_{i}\right) S$ and $\mu_{i}=$ $H_{3}\left(U_{i}, I D_{i}, p k_{i}\right), i \in\{1,2, \cdots, n\}$.

(2) Calculate $f(x)=x_{n}+a_{n-1} x^{n-1}+\cdots+a_{1} x+a_{0}$ and $e=f\left(\mu_{i}\right)$

(3) Compute $m \| \omega=H_{4}(S, e) \oplus C$

(4) Verify if $S=H_{2}(m, \omega) P$ holds. If not, $\mathscr{R}_{i}$ stops the process; otherwise, $\mathscr{R}_{i}$ output the plaintext $m$.

\section{Security Analysis of the Proposed CLAMRE Scheme}

5.1. Security Model. In order to prove the security of the CLAMRE scheme, we take into account of the malicious-butpassive KGC. The robust security model is proposed by Hung et al. [28] in the CLAMRE scheme. Two kinds of adversaries are defined as follows.

Type I adversary $\mathscr{A}_{1}: \mathscr{A}_{1}$ is a malicious outside adversary who can replace the users public key with a value chosen by himself/herself. However, $\mathscr{A}_{1}$ cannot access the master private key of KGC.

Type II adversary $\mathscr{A}_{2}: \mathscr{A}_{2}$ behaves as a honest-but-curious KGC who owns the master key. However it does not allow him/her to replace public key of any user. Define the security of a CLAMRE scheme as a game played between an adversary $\mathscr{A} \in\left\{\mathscr{A}_{1}, \mathscr{A}_{2}\right\}$ and a challenger $\mathscr{C}$. During the game, $\mathscr{A}$ can make the following queries to $\mathscr{C}$.

Create - User query: $\mathscr{C}$ generates private key and public key for the user $U_{i} . \mathscr{C}$ sends the user $U_{i}$ 's public key to $\mathscr{A}$.
Public - Key - Retrieve query: $\mathscr{C}$ returns the matching user $U_{i}$ s public key to $\mathscr{A}$.

Public - Key - Replace query: $\mathscr{C}$ replaces the associated users public key with new public key chosen by himself/herself.

Partial-Private-Key-Extract query: $\mathscr{C}$ sends the users partial private key to $\mathscr{A}$.

Secret - Value - Extract query: $\mathscr{C}$ sends the users secret value to $\mathscr{A}$.

Decryption query: $\mathscr{C}$ decrypts the received ciphertext and sends plaintext to $\mathscr{A}$.

We define the confidentiality of a CLAMRE scheme as the indistinguishability against selective multi-identity chosen ciphertext attack (IND-sMID-CCA). The IND-sMID-CCA game is defined as follows.

Game I. This game is to prove the confidentiality of the CLAMRE scheme.

This game is to prove the confidentiality of the CLAMRE scheme.

Phase 1. In this phase, adversary $\mathscr{A}$ selects $n$ target users with identities $I D_{R_{1}}, I D_{R_{2}}, \cdots, I D_{R_{n}}$ and delivers them to $\mathscr{C}$. $\mathscr{C}$ performs setup to generate system parameters and master key.

In this phase, adversary $\mathscr{A}$ selects $n$ target users with identities $I D_{R_{1}}, I D_{R_{2}}, \cdots, I D_{R_{n}}$ and delivers them to $\mathscr{C} \mathscr{C}$ performs setup to generate system parameters and master key.

Phase 2. $\mathscr{A}$ could adaptively make the aforementioned oracle query but does not allow him/her to make Partial Private - Extract/Public - Key - Replace query with $I D \in$ $\left\{I D_{R_{1}}, I D_{R_{2}}, \cdots, I D_{R_{n}}\right\}$ if he/she is $\mathscr{A}_{1} / \mathscr{A}_{2}$.

$\mathscr{A}$ could adaptively make the aforementioned oracle query but does not allow him/her to make Partial Private - Extract/Public - Key - Replace query with $I D \in$ $\left\{I D_{R_{1}}, I D_{R_{2}}, \cdots, I D_{R_{n}}\right\}$ if he/she is $\mathscr{A}_{1} / \mathscr{A}_{2}$.

Challenge. $\mathscr{A}$ chooses two plaintexts $\left\{m_{0}, m_{1}\right\}$ with the same length, then delivers $\left\{m_{0}, m_{1}\right\}$ to $\mathscr{C} . \mathscr{C}$ randomly selects $\lambda \epsilon$ $\{0,1\}$ and uses $\left\{I D_{R_{1}}, I D_{R_{2}}, \cdots, I D_{R_{n}}\right\}$ and the corresponding public key to encrypt the message $m_{\lambda}$ for generation the ciphertext $C T^{*}$. Then $\mathscr{C}$ sends $C T^{*}$ to $\mathscr{A}$.

$\mathscr{A}$ chooses two plaintexts $\left\{m_{0}, m_{1}\right\}$ with the same length, then delivers $\left\{m_{0}, m_{1}\right\}$ to $\mathscr{C} . \mathscr{C}$ randomly selects $\lambda \in\{0,1\}$ and uses $\left\{I D_{R_{1}}, I D_{R_{2}}, \cdots, I D_{R_{n}}\right\}$ and the corresponding public key to encrypt the message $m_{\lambda}$ for generation the ciphertext $C T^{*}$. Then $\mathscr{C}$ sends $C T^{*}$ to $\mathscr{A}$.

Phase 3. In this phase, $\mathscr{A}$ can make the same queries as he/she does in Phase 2 except that he/she cannot make Decryption query with $C T^{*}$ and $\left\{I D_{R_{1}}, I D_{R_{2}}, \cdots, I D_{R_{n}}\right\}$.

In this phase, $\mathscr{A}$ can make the same queries as he/she does in Phase 2 except that he/she cannot make Decryption query with $C T^{*}$ and $\left\{I D_{R_{1}}, I D_{R_{2}}, \cdots, I D_{R_{n}}\right\}$.

Guess. Finally, $\mathscr{A}$ outputs $\lambda^{\prime} \in\{0,1\}$, that is, his/her guess value about $\lambda$. We say that $\mathscr{A}$ wins the game if $\lambda^{\prime}=\lambda$. The 
advantage is that $\mathscr{A}$ against the CLAMRE scheme is defined by $\operatorname{Adv} v_{C L A M R E}^{I N D-s M I D-C C A}(\mathscr{A})=\left|\operatorname{Pr}\left[\lambda^{\prime}=\lambda\right]-1 / 2\right|$.

Finally, $\mathscr{A}$ outputs $\lambda^{\prime} \in\{0,1\}$, that is, his/her guess value about $\lambda$. We say that $\mathscr{A}$ wins the game if $\lambda^{\prime}=\lambda$. The advantage is that $\mathscr{A}$ against the CLAMRE scheme is defined by $\operatorname{Ad} v_{C L A M R E}^{I N D-s M I D-C C A}(\mathscr{A})=\left|\operatorname{Pr}\left[\lambda^{\prime}=\lambda\right]-1 / 2\right|$.

Definition 1. We say a CLAMRE scheme is IND-sMIDCCA secure if $A d v_{C L A M R E}^{I N D-s M I D-C C A}(\mathscr{A})$ is negligible for any polynomial-time-bounded adversary $\mathscr{A}$.

The receiver anonymity of a CLAMRE scheme is defined by the anonymous indistinguishability against selective identity chosen ciphertext attack (ANON-IND-sID-CCA). The ANON-IND-sID-CCA game is defined as follows.

Game II. This game is to prove the anonymity of the CLAMRE scheme scheme

This game is to prove the anonymity of the CLAMRE

Phase 1. In this phase, $\mathscr{A}$ selects two target users with identities $\left\{I D_{0}, I D_{1}\right\}$ and sends them to $\mathscr{C}$. Then $\mathscr{C}$ runs setup to generate system parameters and the master key.

In this phase, $\mathscr{A}$ selects two target users with identities $\left\{I D_{0}, I D_{1}\right\}$ and sends them to $\mathscr{C}$. Then $\mathscr{C}$ runs setup to generate system parameters and the master key.

Phase 2. In this phase, $\mathscr{A}$ could adaptively make the aforementioned the oracle query. However he/she cannot make Create-User, Secret-Value-Extract/Public-Key-Replace query with $I D \in\left\{I D_{R_{1}}, I D_{R_{2}}, \cdots, I D_{R_{n}}\right\}$ if he/she is $\mathscr{A}_{1} / \mathscr{A}_{2}$.

In this phase, $\mathscr{A}$ could adaptively make the aforementioned the oracle query. However he/she cannot make Create-User, Secret-Value-Extract/Public-Key-Replace query with $I D \in\left\{I D_{R_{1}}, I D_{R_{2}}, \cdots, I D_{R_{n}}\right\}$ if he/she is $\mathscr{A}_{1} / \mathscr{A}_{2}$.

Challenge. $\mathscr{A}$ picks message $m$ together with identities $I D_{R_{2}}^{\prime}, \cdots, I D_{R_{n}}^{\prime}$ and sends them to $\mathscr{C} ; \mathscr{C}$ randomly selects $\lambda \epsilon$ $\{0,1\}$ and uses $\left\{I D_{\lambda}, I D_{R_{2}}^{\prime}, \cdots, I D_{R_{n}}^{\prime}\right\}$ and the corresponding public keys to generate a ciphertext $C T^{*}$ of a message $m_{\lambda}$. Then $\mathscr{C}$ delivers $C T^{*}$ to $\mathscr{A}$.

$\mathscr{A}$ picks message $m$ together with identities $I D_{R_{2}}^{\prime}, \cdots$, $I D_{R_{n}}^{\prime}$ and sends them to $\mathscr{C} ; \mathscr{C}$ randomly selects $\lambda \in\{0,1\}$ and uses $\left\{I D_{\lambda}, I D_{R_{2}}^{\prime}, \cdots, I D_{R_{n}}^{\prime}\right\}$ and the corresponding public keys to generate a ciphertext $C T^{*}$ of a message $m_{\lambda}$. Then $\mathscr{C}$ delivers $C T^{*}$ to $\mathscr{A}$.

Phase 3. In this phase, $\mathscr{A}$ can make the same queries as he/she does in Phase 2 except that he/she cannot make Decryption query with $C T^{*}$ and $\left\{I D_{\lambda}, I D_{R_{2}}^{\prime}, \cdots, I D_{R_{n}}^{\prime}\right\}$.

In this phase, $\mathscr{A}$ can make the same queries as he/she does in Phase 2 except that he/she cannot make Decryption query with $C T^{*}$ and $\left\{I D_{\lambda}, I D_{R_{2}}^{\prime}, \cdots, I D_{R_{n}}^{\prime}\right\}$.

Guess. Finally, $\mathscr{A}$ returns $\lambda^{\prime} \in\{0,1\}$ as his/her guess value about $\lambda$. We say that $\mathscr{A}$ wins the game if $\lambda^{\prime}=\lambda$. The advantage is that $\mathscr{A}$ against the game is defined by $A d v_{C L A M R E}^{A N O N-I N D-s I D-C C A}(\mathscr{A})=\left|\operatorname{Pr}\left[\lambda^{\prime}=\lambda\right]-1 / 2\right|$.

Finally, $\mathscr{A}$ returns $\lambda^{\prime} \in\{0,1\}$ as his/her guess value about $\lambda$. We say that $\mathscr{A}$ wins the game if $\lambda^{\prime}=\lambda$. The advantage is that $\mathscr{A}$ against the game is defined by $A d v_{C L A M R E}^{A N O N-I N D-s I D-C C A}(\mathscr{A})=\left|\operatorname{Pr}\left[\lambda^{\prime}=\lambda\right]-1 / 2\right|$.

Definition 2. We say a CLAMRE scheme is ANON-IND-sIDCCA secure if $A d v_{C L A M R E}^{A N O N-I N D-s I D-C C A}(\mathscr{A})$ is negligible for any polynomial-time-bounded adversary $\mathscr{A}$.

5.2. Security Theorems. In this subsection, we will analyze in detail security of the proposed CLAMRE scheme. Through the analysis, it is shown that the proposed CLAMRE scheme is IND-sMID-CCA secure and ANON-IND-sIDCCA secure against two types of adversaries $\mathscr{A}_{1}, \mathscr{A}_{2}$.

Theorem 3. The proposed CLMRE scheme correctly generates the ciphertext $\sigma=(S, C, a)$, where $a=\left(a_{1}, a_{2}, \cdots, a_{n-1}\right)$ and receiver $\mathscr{R}_{i}(1 \leq i \leq n)$ decrypts it appropriately.

Proof. Due to the fact that $\left(t_{i}+s_{I D_{i}}\right) S=\left(t_{i}+r_{i}+k_{I D_{i}} x\right) s P$ $=s\left(t_{i} P+r_{i} P+k_{I D_{i}} P_{p u b}\right)=s\left(P K_{I D_{i}}+R_{I D_{i}}+k_{I D_{i}} P_{p u b}\right)=U_{i}$, receiver $\mathscr{R}_{i}$ computes the following: $\mu_{i}=H_{3}\left(U_{i}, I D_{i}, p k_{i}\right)$ and $e=f\left(\mu_{i}\right) \cdot m \| \omega=H_{4}(S, e) \oplus C$, and $s=H_{2}(m, \omega)$ is true.

So we proposed that CLAMRE scheme is correct and consistent.

Theorem 4. In the random oracle model, our CLAMRE scheme satisfies the IND-sMID-CCA against the adversary $\mathscr{A}_{1}$ with the hardness assumption of DDH problem.

Proof. $\mathscr{A}_{1}$ is the polynomial time-bounded adversary, if $\mathscr{A}_{1}$ has the ability to break the security of the proposed CLAMRE scheme. Then we can construct a probabilistic polynomial time-bounded challenger $\mathscr{C}$ to solve the $\mathrm{DDH}$ problem by interacting with the adversary $\mathscr{A}_{1}$; that is, given an instance $(P, a P, b P, X)$ of the DDH problem, challenger $\mathscr{C}$ is able to determine if $X=a b \cdot P$ holds. Challenger $\mathscr{C}$ maintains the following initial-empty lists in order to achieve the consistency between queries made by the adversary $\mathscr{A}_{1}$ :

Phase 1

In this phase, $\mathscr{A}_{1}$ selects $n$ target identities; we denote these identities as $I D_{1}, \cdots, I D_{n}$.

$\mathscr{C}$ sets $P_{\text {pub }} \longleftarrow x \cdot P$, and executes setup algorithm to generate other parameters. Then $\mathscr{C}$ delivers $\left\{p, q, l_{1}, l_{2}, E\right.$, $\left.G, G_{q}, P, P_{\text {pub }}, H_{1}, H_{2}, H_{3}, H_{4}\right\}$ to $\mathscr{A}_{1}$. To achieve the random oracles, $\mathscr{C}$ maintains four lists $L_{H_{i}}^{\text {list }}$, where $L_{H_{i}}^{\text {list }}$ is initialized empty ( $i=1,2,3,4)$. The four random oracles make the following answer for $\mathscr{A}_{1} \mathrm{~s}$ queries.

(i) $H_{1}\left(I D_{i}, P K_{I D_{i}}, R_{I D_{i}}\right): \mathscr{C}$ checks if $\left(I D_{i}, P K_{I D_{i}}, R_{I D_{i}}\right.$, $k_{I D_{i}}$ ) exists in $L_{H_{1}}^{\text {list }}$. If so, $\mathscr{C}$ sends $k_{I D_{i}}$ to $\mathscr{A}_{1}$. Otherwise, $\mathscr{C}$ randomly chooses value $k_{I D_{i}} \in Z_{q}^{*}$, inserts $\left(I D_{i}, P K_{I D_{i}}\right.$, $R_{I D_{i}}, k_{I D_{i}}$ ) into $L_{H_{1}}^{\text {list }}$, and sends $k_{I D_{i}}$.

(ii) $H_{2}(m, \omega): \mathscr{C}$ checks if $(m, \omega, s)$ exists in $L_{H_{1}}^{\text {list }}$. If so, $\mathscr{C}$ returns $s$ to $\mathscr{A}_{1}$. Otherwise, $\mathscr{C}$ randomly chooses value $s \in$ $Z_{q}^{*}$, inserts $(m, \omega, s)$ into $L_{H_{2}}^{\text {list }}$, and returns $s$. 
(iii) $H_{3}\left(U_{i}, I D_{i}, p k_{i}\right): \mathscr{C}$ checks if $\left(U_{i}, I D_{i}, p k_{i}, \mu_{i}\right)$ exists in $L_{H_{3}}^{\text {list }}$. If so, $\mathscr{C}$ returns $\mu_{i}$ to $\mathscr{A}_{1}$. Otherwise, $\mathscr{C}$ randomly chooses value $\mu_{i} \in Z_{q}^{*}$, inserts $\left(U_{i}, I D_{i}, p k_{i}, \mu_{i}\right)$ into $L_{H_{3}}^{\text {list }}$, and returns $\mu_{i}$.

(iv) $H_{4}(S, e): \mathscr{C}$ checks if $(S, e, \tau)$ exists in $L_{H_{4}}^{\text {list }}$. If so, $\mathscr{C}$ returns $\tau$ to $\mathscr{A}_{1}$. Otherwise, $\mathscr{C}$ randomly selects an element $\tau \in\{0,1\}^{l_{1}+l_{2}}$, inserts $(S, e, \tau)$ into $L_{H_{4}}^{\text {list }}$, and returns $\tau$.

Phase 2

$\mathscr{A}_{1}$ can adaptively make queries to $\mathscr{C} . \mathscr{C}$ maintains a list $L_{R}^{\text {list }}$, which is initialized empty. Challenger $\mathscr{C}$ responded to these queries made by adversary $\mathscr{A}_{1}$ as follows.

(i) Create - User $\left(I D_{\mathscr{R}_{i}}\right)$ query: $\mathscr{C}$ checks if $\left(I D_{\mathscr{R}_{i}}, r_{i}\right.$, $\left.s_{I D_{i}}, t_{i}, R_{I D_{i}}, P K_{I D_{i}}\right)$ exists in $L_{R}^{\text {list }}$. If so, $\mathscr{C}$ returns $\left(R_{I D_{i}}, P K_{I D_{i}}\right)$ to $\mathscr{A}_{1}$. Otherwise, $\mathscr{C}$ executes the following processes.

(ii) If $I D_{\mathscr{R}_{i}} \in\left\{I D_{1}, \cdots, I D_{n}\right\}$ holds, without losing generality, we suppose $I D_{\mathscr{R}_{i}}=I D_{i}, \mathscr{C}$ randomly picks $r_{i}, t_{i} \in$ $Z_{q}^{*}$, computes $P K_{I D_{i}}=t_{i} P, R_{I D_{i}}=r_{i} a P, k_{I D_{i}}=H_{1}\left(I D_{i}, P K_{I D_{i}}\right.$, $\left.R_{I D_{i}}\right)$, and sets $s_{I D_{i}} \longleftarrow \perp . \mathscr{C}$ inserts $\left(I D_{i}, P K_{I D_{i}}, R_{I D_{i}}, k_{I D_{i}}\right)$ and $\left(I D_{i}, r_{i}, s_{I D_{i}}, t_{i}, R_{I D_{i}}, P K_{I D_{i}}\right)$ into $L_{H_{1}}^{\text {list }}$ and $L_{R}^{\text {list }}$, respectively. At last, $\mathscr{C}$ returns $\left(R_{I D}, P K_{I D_{i}}\right)$ to $\mathscr{A}_{1}$.

(iii) Otherwise $I D_{\mathscr{R}_{i}} \notin\left\{I D_{1}, \cdots, I D_{n}\right\} ; \mathscr{C}$ randomly picks $t_{i}, s_{I D_{i}}, k_{I D_{i}} \in Z_{q}^{*}$ and computes $R_{I D_{i}}=s_{I D_{i}} P-k_{I D_{i}} P_{\text {pub }}$, $P K_{I D_{i}}=t_{i} P . \mathscr{C}$ inserts $\left(I D_{i}, P K_{I D_{i}}, R_{I D_{i}}, k_{I D_{i}}\right)$ and $\left(I D_{i}, r_{i}\right.$, $\left.s_{I D_{i}}, t_{i}, R_{I D_{i}}, P K_{I D_{i}}\right)$ into $L_{H_{1}}^{\text {list }}$ and $L_{R}^{\text {list }}$, respectively. At last, $\mathscr{C}$ returns $\left(R_{I D_{i}}, P K_{I D_{i}}\right)$ to $\mathscr{A}_{1}$.

(iv) Public - Key-Retrieve $\left(I D_{R_{i}}\right): \mathscr{C}$ checks if $\left(I D_{R_{i}}\right.$, $\left.s_{I D_{i}}, t_{i}, R_{I D_{i}}, P K_{I D_{i}}\right)$ exists in $L_{R}^{\text {list }}$. If not, $\mathscr{C}$ makes the CreateUser query with $I D_{i}$ first. Then, $\mathscr{C}$ returns $\left(R_{I D_{i}}, P K_{I D_{i}}\right)$ to $\mathscr{A}_{1}$.

(v) Public - key - Replace $\left(I D_{R_{i}}, R_{I D_{i}}^{\prime}, P K_{I D_{i}}^{\prime}\right)$ : $\mathscr{C}$ checks if $\left(I D_{R_{i}}, s_{I D_{i}}, t_{i}, R_{I D_{i}}, P K_{I D_{i}}\right)$ exists in $L_{R}^{\text {list }}$. If not, $\mathscr{C}$ makes the Create-User query with $I D_{i}$ first. Then, $\mathscr{C}$ replaces $\left(R_{I D_{i}}, P K_{I D_{i}}\right)$ with $\left(I D_{i}, R_{I D_{i}}^{\prime}, P K_{I D_{i}}^{\prime}\right)$

(vi) Partial - Private - Key - Extract $\left(I D_{R_{i}}\right): \mathscr{C}$ checks if $\left(I D_{R_{i}}, s_{I D_{i}}, t_{i}, R_{I D_{i}}, P K_{I D_{i}}\right)$ exists in $L_{R}^{\text {list }}$. If not, $\mathscr{C}$ makes the Create-User query with $I D_{R_{i}}$ first. Then, $\mathscr{C}$ returns $s_{I D_{i}}$ to $\mathscr{A}_{1}$.

(vii) Secret - Value - Extract $\left(I D_{R_{i}}\right): \mathscr{C}$ checks if $\left(I D_{R_{i}}, s_{I D_{i}}, t_{i}, R_{I D_{i}}, P K_{I D_{i}}\right)$ exists in $L_{R}^{\text {list }}$. If not, $\mathscr{C}$ makes the Create-User query with $I D_{R_{i}}$ first. Then, $\mathscr{C}$ returns $t_{i}$ to $\mathscr{A}_{1}$.

(viii) Decryption $\left(I D_{R_{i}}, \sigma_{i}\right): \mathscr{C}$ checks if $I D_{\mathscr{R}_{i}} \in$ $\left\{I D_{1}, \cdots, I D_{n}\right\}$ holds, where $\sigma_{i}=\left(S_{i}, C_{i}, f_{i}\right)$. If not, $\mathscr{C}$ looks up $L_{R}^{\text {list }}$ for $\left(I D_{R_{i}}, s_{I D_{i}}, t_{i}, R_{I D_{i}}, P K_{I D_{i}}\right)$ and uses $\left(s_{I D_{i}}, t_{i}\right)$ to decrypt the ciphertext. Otherwise $I D_{\mathscr{R}_{i}} \in\left\{I D_{1}, \cdots, I D_{n}\right\}, \mathscr{C}$ responds according to the following steps.

(ix) $\mathscr{C}$ looks up $L_{H_{4}}^{\text {list }}$ for $\left(S_{i}, e_{i}, \tau_{i}\right)$. If not, $\mathscr{C}$ outputs failure and stops.

(x) $\mathscr{C}$ searches the tuple $\left(m_{i}, \omega_{i}, s_{i}\right)$ from $L_{H_{2}}^{\text {list }}$ and checks if $S_{i}=s_{i} P$ holds. If so, $\mathscr{C}$ keeps $\left(m_{i}, \omega_{i}\right)$; if not, $\mathscr{C}$ outputs failure and stops.

(xi) $\mathscr{C}$ checks if $C_{i}=\tau_{i} \oplus\left(m_{i} \| \omega_{i}\right)$ holds. If not, $\mathscr{C}$ outputs failure and stops. Otherwise return $m_{i}$ to $\mathscr{A}_{1}$

Challenge. After making the above queries, $\mathscr{A}_{1}$ picks two messages $m_{0}$ and $m_{1}$ with length $l_{2}$ and sends them to challenger $\mathscr{C}, \mathscr{C}$ chooses $\lambda \in\{0,1\}$ at random and performs the following steps. (i) $\mathscr{C}$ sets $S^{*} \longleftarrow b \cdot P$.

(ii) Let $U_{i}=\left(k_{I D_{i}} x+t_{i}\right) S^{*}+r_{i} X$, and compute $\mu_{i}=$ $H_{3}\left(U_{i}, I D_{i}, p k_{i}\right), i=1,2, \cdots, n$

(iii) $\mathscr{C}$ chooses $e \in Z_{q}^{*}$ at random and computes a polynomial $f(x)$ with degree $n$ as follows:

$$
\begin{aligned}
f(x) & =\prod_{i=1}^{n}\left(x-\mu_{i}\right)+e(\bmod q) \\
= & x^{n}+a_{n-1}^{*} x^{n-1}+\cdots+a_{1}^{*} x+a_{0}^{*}, \\
& \quad \text { where } a_{i} \in Z_{q}^{*}(i=0,1, \ldots, n-1)
\end{aligned}
$$

(iv) $\mathscr{C}$ chooses $\tau^{*} \in\{0,1\}^{l_{1}+1_{2}}$ and $\omega^{*} \in\{0,1\}^{l_{2}}$ at random and computes $C^{*}=\tau^{*} \oplus\left(m_{\lambda} \| \omega^{*}\right)$.

Final, $\mathscr{C}$ sends the ciphertext $\sigma^{*}=\left(S^{*}, C^{*}, f^{*}\right)$.

Phase 3

In this phase, $\mathscr{A}_{1}$ can make the same queries in Phase 2 except that it cannot make decryption queries with $I D^{*} \in$ $\left\{I D_{1}, I D_{2}, \cdots, I D_{n}\right\}$ and $\sigma^{*}$.

Guess

$\mathscr{A}_{1}$ outputs $\lambda^{\prime} \in\{0,1\}$ as his/her guess value about $\lambda$. If $\lambda=\lambda^{\prime}$, then $\mathscr{C}$ outputs 1 ; otherwise, $\mathscr{C}$ outputs $0 . \mathscr{A}_{1}$ wins the game if and only if $\lambda=\lambda^{\prime}$ holds.

Based on the above oracle queries, the simulation of $\mathscr{C}$ is perfect. Next, we consider the probability that challenger $\mathscr{C}$ fails in Game I. Combined with the previous description, we know that $\mathscr{C}$ fails in Decryption query if $\left(S_{i}, e_{i}\right)$ is not in $L_{H_{4}}^{\text {list }}$. The probability that $\mathscr{A}_{1}$ can correctly guess the output of $H_{4}$ : $\{0,1\}^{*} \longrightarrow\{0,1\}^{l_{1}+l_{2}}$ is $1 / 2^{l_{1}+l_{2}}$. Therefore, the probability of $\mathscr{C}$ failure in game I is less than $q_{d} / 2^{l_{1}+l_{2}}$, where $q_{d}$ denote the decryption query times in the game.

If $X=a b P$ holds, then $\sigma^{*}$ is valid ciphertext. Thus, $\mathscr{A}_{1}$ is able to distinguish $\lambda$ with nonnegligible advantage $\epsilon$.

$$
\begin{aligned}
\operatorname{Pr}[c=1 \mid X=a b P] & =\operatorname{Pr}\left[\lambda=\lambda^{\prime} \mid X=a b P\right] \\
& =\frac{1}{2}+\epsilon .
\end{aligned}
$$

If $X \neq a b P$, then the ciphertext distribution is random and uniform when $\lambda=0$ or $\lambda=1$, so $\mathscr{A}_{1}$ cannot distinguish $\lambda$ with any advantage.

$$
\operatorname{Pr}[c=1 \mid X \neq a b P]=\operatorname{Pr}\left[\lambda=\lambda^{\prime} \mid X \neq a b P\right]=\frac{1}{2} .
$$

Therefore, if $\mathscr{A}_{1}$ can break the IND-sMID-CCA security of the proposed CLAMRE scheme with nonnegligible advantage $\epsilon$, then challenger $\mathscr{C}$ can solve the DDH problem with a nonnegligible advantage $\epsilon-q_{d} / 2^{l_{1}+l_{2}}$, because the $\mathrm{DDH}$ problem is difficult. Therefore, the proposed CLAMRE scheme is IND-sMID-CCA secure against $\mathscr{A}_{1}$.

Theorem 5. Our CLAMRE scheme is IND-sMID-CCA secure against type II adversary $\mathscr{A}_{2}$ under random oracle model with the difficulties of computational Diffie-Hellman problem.

Proof. $\mathscr{A}_{2}$ is the polynomial time-bounded adversary, if $\mathscr{A}_{2}$ has the ability to break the security of the proposed CLAMRE 
scheme. Then we can construct a probabilistic polynomial time-bounded challenger $\mathscr{C}$ to solve the DDH problem by interacting with the adversary $\mathscr{A}_{2}$; that is, for given an instance $(P, a P, b P, X)$ of the $\mathrm{DDH}$ problem, challenger $\mathscr{C}$ is able to determine if $X=a b \cdot P$ holds. Challenger $\mathscr{C}$ maintains the following initial-empty lists in order to achieve the consistency between queries made by adversary $\mathscr{A}_{2}$.

Phase 1

In this phase, $\mathscr{A}_{2}$ selects $n$ target identities; we denote these identities as $I D_{1}, \cdots, I D_{n}$.

$\mathscr{C}$ picks $x \in Z_{q}^{*}$ at random as system private key and computes corresponding public key $P_{p u b}=x \cdot P . \mathscr{C}$ performs Setup algorithm to construct other parameters. At last, $\mathscr{C}$ delivers $\left\{p, q, l_{1}, l_{2}, E, G, G_{q}, P, P_{p u b}, H_{1}, H_{2}, H_{3}, H_{4}\right\}$ to $\mathscr{A}_{2}$ and master private key $x$ to $\mathscr{A}_{2}$. To achieve the random oracles, $\mathscr{C}$ maintains four lists $L_{H_{i}}^{\text {list }}$, where initial $L_{H_{i}}^{\text {list }}$ is empty $(i=1,2,3,4)$. The four random oracles make the following answer for $\mathscr{A}_{2} s$ queries.

(i) $H_{1}\left(I D_{i}, P K_{I D_{i}}, R_{I D_{i}}\right): \mathscr{C}$ checks if $\left(I D_{i}, P K_{I D_{i}}, R_{I D_{i}}\right.$, $k_{I D_{i}}$ ) exists in $L_{H_{1}}^{\text {list }}$. If so, $\mathscr{C}$ returns $k_{I D_{i}}$ to $\mathscr{A}_{2}$. Otherwise, $\mathscr{C}$ randomly selects an element $k_{I D_{i}} \in Z_{q}^{*}$, inserts $\left(I D_{i}, P K_{I D_{i}}\right.$, $R_{I D_{i}}, k_{I D_{i}}$ ) into $L_{H_{1}}^{\text {list }}$, and returns $k_{I D_{i}}$.

(ii) $H_{2}(m, \omega): \mathscr{C}$ checks if $(m, \omega, s)$ exists in $L_{H_{1}}^{\text {list }}$. If so, $\mathscr{C}$ returns $s$ to $\mathscr{A}_{2}$. Otherwise, $\mathscr{C}$ randomly selects an element $s \in Z_{q}^{*}$, inserts $(m, \omega, s)$ into $L_{H_{2}}^{\text {list }}$, and returns $s$.

(iii) $H_{3}\left(U_{i}, I D_{i}, p k_{i}\right): \mathscr{C}$ checks if $\left(U_{i}, I D_{i}, p k_{i}, \mu_{i}\right)$ exists in $L_{H_{3}}^{\text {list }}$. If so, $\mathscr{C}$ returns $\mu_{i}$ to $\mathscr{A}_{2}$. Otherwise, $\mathscr{C}$ randomly selects an element $\mu_{i} \in Z_{q}^{*}$, inserts $\left(U_{i}, I D_{i}, p k_{i}, \mu_{i}\right)$ into $L_{H_{3}}^{\text {list }}$, and returns $\mu_{i}$.

(iv) $H_{4}(S, e): \mathscr{C}$ checks if $(S, e, \tau)$ exists in $L_{H_{4}}^{\text {list }}$. If so, $\mathscr{C}$ returns $\tau$ to $\mathscr{A}_{2}$. Otherwise, $\mathscr{C}$ randomly selects an element $\tau \in\{0,1\}^{l_{1}+l_{2}}$, inserts $(S, e, \tau)$ into $L_{H_{4}}^{\text {list }}$, and returns $\tau$.

Phase 2

In this phase, $\mathscr{A}_{2}$ can adaptively make a lot of queries to $\mathscr{C} . \mathscr{C}$ maintains a list $L_{R}^{\text {list }}$, which is initialized empty. These queries are responded as follows.

(i) Create - User $\left(I D_{\mathscr{R}_{i}}\right)$ query: $\mathscr{C}$ checks if $\left(I D_{\mathscr{R}_{i}}, r_{i}\right.$, $\left.s_{I D_{i}}, t_{i}, R_{I D_{i}}, P K_{I D_{i}}\right)$ exists in $L_{R}^{\text {list }}$. If so, $\mathscr{C}$ returns $\left(R_{I D_{i}}, P K_{I D_{i}}\right)$ to $\mathscr{A}_{2}$. Otherwise, $\mathscr{C}$ performs the following steps.

(ii) If $I D_{\mathscr{R}_{i}} \in\left\{I D_{1}, \cdots, I D_{n}\right\}$ holds, without losing generality, we suppose $I D_{\mathscr{R}_{i}}=I D_{i}, \mathscr{C}$ randomly chooses $r_{i}, t_{i} \in Z_{q}^{*}$ and calculates $P K_{I D_{i}}=t_{i} \cdot a P, R_{I D_{i}}=r_{i} P, k_{I D_{i}}=H_{1}\left(P K_{I D_{i}}\right.$, $\left.R_{i}, I D_{i}\right), s_{I D_{i}}=r_{i}+k_{I D_{i}} x \bmod q . \mathscr{C}$ inserts $\left(I D_{i}, P K_{I D_{i}}, R_{I D_{i}}\right.$, $\left.k_{I D_{i}}\right)$ and $\left(I D_{i}, r_{i}, s_{I D_{i}}, t_{i}, R_{I D_{i}}, P K_{I D_{i}}\right)$ into $L_{1}^{\text {list }}$ and $L_{R}^{\text {list }}$, respectively. At last, $\mathscr{C}$ returns $\left(R_{I D_{i}}, P K_{I D_{i}}\right)$ to $\mathscr{A}_{2}$.

(iii) Otherwise $I D_{\mathscr{R}_{i}} \notin\left\{I D_{1}, \cdots, I D_{n}\right\} ; \mathscr{C}$ randomly picks $t_{i}, r_{i}, k_{I D_{i}} \in Z_{q}^{*}$ and computes $R_{I D_{i}}=r_{i} P, s_{I D_{i}}=r_{i}+k_{I D_{i}} s \bmod$ $q, P K_{I D_{i}}=t_{i} \cdot P . \mathscr{C}$ inserts $\left(I D_{i}, P K_{I D_{i}}, R_{I D_{i}}, k_{I D_{i}}\right)$ and $\left(I D_{i}, r_{i}\right.$, $\left.s_{I D_{i}}, t_{i}, R_{I D_{i}}, P K_{I D_{i}}\right)$ into $L_{1}^{\text {list }}$ and $L_{R}^{\text {list }}$, respectively. At last, $\mathscr{C}$ returns $\left(R_{I D_{i}}, P K_{I D_{i}}\right)$ to $\mathscr{A}_{2}$.

(iv) Public - Key-Retrieve $\left(I D_{R_{i}}\right): \mathscr{C}$ checks if $\left(I D_{R_{i}}, s_{I D_{i}}\right.$, $t_{i}, R_{I D_{i}}, P K_{I D_{i}}$ ) exists in $L_{R}^{\text {list }}$. If not, $\mathscr{C}$ makes the Create-User query with $I D_{i}$ first. Then, $\mathscr{C}$ returns $R_{I D_{i}}, P K_{I D_{i}}$ to $\mathscr{A}_{2}$.

(v) Partial - Private - Key - Extract $\left(I D_{R_{i}}\right): \mathscr{C}$ checks if $\left(I D_{R_{i}}, s_{I D_{i}}, t_{i}, R_{I D_{i}}, P K_{I D_{i}}\right)$ exists in $L_{R}^{\text {list }}$. If not, $\mathscr{C}$ makes the
Create-User query with $I D_{R_{i}}$ first. Then, $\mathscr{C}$ returns $s_{I D_{i}}$ to $\mathscr{A}_{2}$.

(vi) Secret - Value - Extract $\left(I D_{R_{i}}\right): \mathscr{C}$ checks if $\left(I D_{R_{i}}, s_{I D_{i}}, t_{i}, R_{I D_{i}}, P K_{I D_{i}}\right)$ exists in $L_{R}$. If not, $\mathscr{C}$ makes the Create-User query with $I D_{R_{i}}$ first. Then, $\mathscr{C}$ returns $t_{i}$ to $\mathscr{A}_{1}$.

(vii) Decryption $\left(I D_{R_{1}}, \sigma_{i}\right): \mathscr{C}$ checks if $I D_{\mathscr{R}_{i}} \in\left\{I D_{1}, \cdots\right.$, $\left.I D_{n}\right\}$ holds, where $\sigma_{i}=\left(S_{i}, C_{i}, a_{i}\right), a_{i}=\left(a_{i 0}, a_{i 1}, \cdots, a_{i(n-1)}\right)$. If not, $\mathscr{C}$ looks up $L_{R}^{\text {list }}$ for $\left(I D_{R_{i}}, s_{I D_{i}}, t_{i}, R_{I D_{i}}, P K_{I D_{i}}\right)$ and uses $\left(s_{I D_{i}}, t_{i}\right)$ to decrypt the ciphertext. Otherwise $I D_{\mathscr{R}_{i}} \in$ $\left\{I D_{1}, \cdots, I D_{n}\right\}, \mathscr{C}$ responds according to the following steps.

(viii) $\mathscr{C}$ looks up $L_{4}^{\text {list }}$ for $\left(S_{i}, e_{i}, \tau_{i}\right)$. If not, $\mathscr{C}$ outputs failure and stops.

(ix) $\mathscr{C}$ searches the tuple $\left(m_{i}, \omega_{i}, s_{i}\right)$ from $L_{H_{2}}^{\text {list }}$ and checks if $S_{i}=s_{i} P$ holds. If so, $\mathscr{C}$ keeps $\left(m_{i}, \omega_{i}\right)$; if not, $\mathscr{C}$ outputs failure and stops.

(x) $\mathscr{C}$ checks if $C_{i}=\tau_{i} \oplus\left(m_{i} \| \omega_{i}\right)$ holds. If not, $\mathscr{C}$ outputs failure and stops. Otherwise, return $m_{i}$ to $\mathscr{A}_{2}$

Challenge

After making the above queries, $\mathscr{A}_{2}$ picks two messages $m_{0}$ and $m_{1}$ with length $l_{2}$ and sends them to challenger $\mathscr{C} ; \mathscr{C}$ chooses $\lambda \in\{0,1\}$ at random and implements the following process.

(i) $\mathscr{C}$ sets $S^{*} \longleftarrow b \cdot P$.

(ii) Let $U_{i}=\left(k_{I D_{i}} x+r_{i}\right) S^{*}+t_{i} \cdot X$, and compute $\mu_{i}=$ $H_{3}\left(U_{i}, I D_{i}, p k_{i}\right) . i=1,2, \cdots, n$

(iii) $\mathscr{C}$ chooses $e \in Z_{q}^{*}$ at random and computes a polynomial $f(x)$ with degree $n$ as follows:

$$
\begin{aligned}
f(x)= & \prod_{i=1}^{n}\left(x-\mu_{i}\right)+e(\bmod q) \\
= & x^{n}+a_{n-1}^{*} x^{n-1}+\cdots+a_{1}^{*} x+a_{0}^{*}, \\
& \quad \text { where } a_{i} \in Z_{q}^{*}(i=0,1, \ldots, n-1)
\end{aligned}
$$

(iv) $\mathscr{C}$ chooses $\tau^{*} \in\{0,1\}^{l_{1}+1_{2}}$ and $\omega^{*} \in\{0,1\}^{l_{2}}$ at random and computes $C^{*}=\tau^{*} \oplus\left(m_{\lambda} \| \omega^{*}\right)$.

Final, $\mathscr{C}$ sends the ciphertext $\sigma^{*}=\left(S^{*}, C^{*}, f^{*}\right)$.

Phase 3

In this phase, $\mathscr{A}_{2}$ can make the same queries in Phase 2 except that he cannot make decryption queries with $I D^{*} \epsilon$ $\left\{I D_{1}, I D_{2}, \cdots, I D_{n}\right\}$ and $\sigma^{*}$.

Guess

$\mathscr{A}_{2}$ outputs $\lambda^{\prime} \in\{0,1\}$ as his/her guess value about $\lambda$. If $\lambda=\lambda^{\prime}$, then $\mathscr{C}$ outputs 1; otherwise, $\mathscr{C}$ outputs $0 . \mathscr{A}_{2}$ wins the game if and only if $\lambda=\lambda^{\prime}$ holds.

According to the above oracle queries, we know that the simulation of $\mathscr{C}$ is perfect. Now, we analyze the probability that $\mathscr{C}$ fails in Game I. Based on the above description, we know that $\mathscr{C}$ fails in decryption query if $\left(S_{i}, e_{i}\right)$ is not in $L_{4}^{\text {list }}$. The probability that $\mathscr{A}_{2}$ can correctly guess the output of $H_{4}$ : $\{0,1\}^{*} \longrightarrow\{0,1\}^{l_{1}+l_{2}}$ is $1 / 2^{l_{1}+l_{2}}$. Therefore, the probability that $\mathscr{C}$ fails in the game is less than $q_{d} / 2^{l_{1}+l_{2}}$, where $q_{d}$ denotes the decryption queries involved in the game.

If $X=a b P$ holds, then $\sigma^{*}$ is valid ciphertext. Thus, $\mathscr{A}_{1}$ is able to distinguish $\lambda$ with nonnegligible advantage $\epsilon$.

$$
\operatorname{Pr}[c=1 \mid X=a b P]=\operatorname{Pr}\left[\lambda=\lambda^{\prime} \mid X=a b P\right]
$$




$$
=\frac{1}{2}+\epsilon
$$

If $X \neq a b P$, then the ciphertext distribution is random and uniform when $\lambda=0 \operatorname{or} \lambda=1$. So $\mathscr{A}_{2}$ cannot distinguish $\lambda$ with any advantage.

$$
\operatorname{Pr}[c=1 \mid X \neq a b P]=\operatorname{Pr}\left[\lambda=\lambda^{\prime} \mid X \neq a b P\right]=\frac{1}{2} .
$$

Therefore, if $\mathscr{A}_{2}$ can break the IND-sMID-CCA security of the proposed CLAMRE scheme with nonnegligible advantage $\epsilon$, then challenger $\mathscr{C}$ can solve the DDH problem with a nonnegligible advantage $\epsilon-q_{d} / 2^{l_{1}+l_{2}}$. If $\mathscr{A}_{2}$ can break the IND-sMID-CCA security of the proposed CLAMRE scheme with nonnegligible advantage $\epsilon$, then we know $\mathscr{C}$ can solve the $\mathrm{CDH}$ problem with a nonnegligible advantage $\epsilon-q_{d} / 2^{l_{1}+l_{2}}$. Due to the fact that the $\mathrm{CDH}$ problem is hard, we know that the proposed CLAMRE scheme is IND-sMID-CCA secure against adversary $\mathscr{A}_{2}$.

Theorem 6. In the random oracle model, our proposed CLAMRE scheme is ANON-IND-sID-CCA secure against adversary $\mathscr{A}_{1}$ with the difficulty assumption of $\mathrm{DDH}$ problem.

Proof. Assume that the adversary $\mathscr{A}_{1}$ can breach our CLAMRE scheme, then we will be able to design a challenger $\mathscr{C}$ for solving an instance of DDH problem; that is, for given an instance $(P, a \cdot P, b \cdot P, X)$ of DDH problem, challenger $\mathscr{C}$ can determine if $X=a b P$ holds by interacting with adversary $\mathscr{A}_{1}$. Similar to Theorem 4 , let lists $L_{H_{i}}^{\text {list }}(i=1,2,3,4)$ and $L_{R}^{\text {list }}$ be maintained by challenger $\mathscr{C}$.

Phase 1. Assume that adversary $\mathscr{A}_{1}$ selects two target users with identities $I D_{0}, I D_{1}$. Challenger $\mathscr{C}$ randomly selects $\lambda \epsilon$ $\{0,1\}$

Assume that adversary $\mathscr{A}_{1}$ selects two target users with identities $I D_{0}, I D_{1}$. Challenger $\mathscr{C}$ randomly selects $\lambda \in\{0,1\}$

$\mathscr{C}$ sets $P_{\text {pub }} \longleftarrow x \cdot P$, and implements Setup algorithm to construct other parameters. At last, $\mathscr{C}$ delivers $\left\{p, q, l_{1}, l_{2}, E, G, G_{q}, P, P_{p u b}, H_{1}, H_{2}, H_{3}, H_{4}\right\}$ to $\mathscr{A}_{1}$. Challenger $\mathscr{C}$ returns answers to the adversary $\mathscr{A}_{1}$ s queries in the following ways.

Hash queries to $H_{i}(i=1,2,3,4)$ : these queries are the same as those performed in Theorem 4 .

Phase 2. Now, challenger $\mathscr{C}$ will respond to the queries made by the adversary $\mathscr{A}_{1}$ in the following ways.

Now, challenger $\mathscr{C}$ will respond to the queries made by the adversary $\mathscr{A}_{1}$ in the following ways.

(i) Create - User $\left(I D_{\mathscr{R}_{i}}\right)$ query: $\mathscr{C}$ checks if $\left(I D_{\mathscr{R}_{1}}, r_{i}\right.$, $\left.s_{I D_{i}}, t_{i}, R_{I D_{i}}, P K_{I D_{i}}\right)$ exists in $L_{R}^{\text {list }}$. If so, $\mathscr{C}$ returns $\left(R_{I D_{i}}, P K_{I D_{i}}\right)$ to $\mathscr{A}_{1}$. Otherwise, $\mathscr{C}$ executes the following processes.

(ii) If $I D_{\mathscr{R}_{i}}=I D_{j}$ for $j \in\{0,1\}$ holds, $\mathscr{C}$ randomly chooses $r_{i}, t_{i} \in Z_{q}^{*}$, computes $P K_{I D_{i}}=t_{i} \cdot P, R_{I D_{i}}=r_{i}$. $a P, k_{I D_{i}}=H_{1}\left(I D_{i}, P K_{I D_{i}}, R_{I D_{i}}\right)$, and sets $s_{I D_{i}} \longleftarrow \perp \mathscr{C}$ inserts
$\left(I D_{i}, R_{I D_{i}}, k_{I D_{i}}\right)$ and $\left(I D_{i}, r_{i}, s_{I D_{i}}, t_{i}, R_{I D_{i}}, P K_{I D_{i}}\right)$ into $L_{H_{1}}^{\text {list }}$ and $L_{R}^{\text {list }}$, respectively. At last, $\mathscr{C}$ returns $\left(R_{I D_{i}}, P K_{I D_{i}}\right)$ to $\mathscr{A}_{1}$.

(iii) Otherwise $I D_{\mathscr{R}_{i}} \notin\left\{I D_{0}, I D_{1}\right\} ; \mathscr{C}$ randomly picks $t_{i}, s_{I D_{i}}, k_{I D_{i}} \in Z_{q}^{*}$ and computes $R_{I D_{i}}=s_{I D_{i}} P-k_{I D_{i}} P_{p u b}$, $P K_{I D_{i}}=t_{i} P . \mathscr{C}$ inserts $\left(I D_{i}, R_{I D_{i}}, k_{I D_{i}}\right)$ and $\left(I D_{i}, r_{i}, s_{I D_{i}}, t_{i}\right.$, $\left.R_{I D_{i}}, P K_{I D_{i}}\right)$ into $L_{1}^{\text {list }}$ and $L_{R}^{\text {list }}$, respectively. At last, $\mathscr{C}$ returns $\left(R_{I D_{i}}, P K_{I D_{i}}\right)$ to $\mathscr{A}_{1}$.

(iv) Public - Key-Retrieve $\left(I D_{R_{i}}\right): \mathscr{C}$ checks if $\left(I D_{R_{i}}, s_{I D_{i}}\right.$, $t_{i}, R_{I D_{i}}, P K_{I D_{i}}$ ) exists in $L_{R}^{\text {list }}$. If not, $\mathscr{C}$ makes the Create-User query with $I D_{i}$ first. Then, $\mathscr{C}$ returns $\left(R_{I D_{i}}, P K_{I D_{i}}\right)$ to $\mathscr{A}_{1}$.

(v) Public - key - Replace $\left(I D_{R_{i}}, R_{I D_{i}}^{\prime}, P K_{I D_{i}}^{\prime}\right): \mathscr{C}$ checks if $\left(I D_{R_{i}}, s_{I D_{i}}, t_{i}, R_{I D_{i}}, P K_{I D_{i}}\right)$ exists in $L_{R}^{\text {list }}$. If not, $\mathscr{C}$ makes the Create-User query with $I D_{i}$ first. Then, $\mathscr{C}$ replaces $\left(R_{I D_{i}}, P K_{I D_{i}}\right)$ with $\left(I D_{i}, R_{I D_{i}}^{\prime}, P K_{I D_{i}}^{\prime}\right)$.

(vi) Partial - Private - Key - Extract $\left(I D_{R_{i}}\right): \mathscr{C}$ checks if $\left(I D_{R_{i}}, s_{I D_{i}}, t_{i}, R_{I D_{i}}, P K_{I D_{i}}\right)$ exists in $L_{R}^{\text {list }}$. If not, $\mathscr{C}$ makes the Create-User query with $I D_{R_{i}}$ first. Then, $\mathscr{C}$ returns $s_{I D_{i}}$ to $\mathscr{A}_{1}$.

(vii) Secret - Value - Extract $\left(I D_{R_{i}}\right): \mathscr{C}$ checks if $\left(I D_{R_{i}}, s_{I D_{i}}, t_{i}, R_{I D_{i}}, P K_{I D_{i}}\right)$ exists in $L_{R}^{\text {list }}$. If not, $\mathscr{C}$ makes the Create-User query with $I D_{R_{i}}$ first. Then, $\mathscr{C}$ returns $t_{i}$ to $\mathscr{A}_{1}$.

(viii) Decryption $\left(I D_{R_{i}}, \sigma_{i}\right): \mathscr{C}$ checks if $I D_{\mathscr{R}_{i}} \in\left\{I D_{0}, I D_{1}\right\}$ holds, where $\sigma_{i}=\left(S_{i}, C_{i}, f_{i}\right)$. If not, $\mathscr{C}$ looks up $L_{R}^{\text {list }}$ for $\left(I D_{R_{i}}, s_{I D_{i}}, t_{i}, R_{I D_{i}}, P K_{I D_{i}}\right)$ and uses full private key $\left(s_{I D_{i}}, t_{i}\right)$ to decrypt the ciphertext. Otherwise $I D_{\mathscr{R}_{i}} \in\left\{I D_{0}, I D_{1}\right\}, \mathscr{C}$ responds as follows.

(ix) $\mathscr{C}$ looks up $L_{H_{4}}^{\text {list }}$ for $\left(S_{i}, e_{i}, \tau_{i}\right)$. If not, $\mathscr{C}$ outputs failure and stops.

(x) $\mathscr{C}$ searches the tuple $\left(m_{i}, \omega_{i}, s_{i}\right)$ from $L_{H_{2}}^{l i s t}$ and checks if $S_{i}=s_{i} P$ holds. If so, $\mathscr{C}$ keeps $\left(m_{i}, \omega_{i}\right)$; if not, $\mathscr{C}$ outputs failure and stops.

(xi) $\mathscr{C}$ checks if $C_{i}=\tau_{i} \oplus\left(m_{i} \| \omega_{i}\right)$ holds. If not, $\mathscr{C}$ outputs failure and stops. Otherwise return $m_{i}$ to $\mathscr{A}_{1}$

Challenge. After making the above queries, $\mathscr{A}_{1}$ picks plaintext $m$ together with identities $\left\{I D_{2}, \cdots, I D_{n}\right\}$ on which he wants to be challenged. $\mathscr{C}$ chooses $\lambda \in\{0,1\}$ at random and implements the following process.

After making the above queries, $\mathscr{A}_{1}$ picks plaintext $m$ together with identities $\left\{I D_{2}, \cdots, I D_{n}\right\}$ on which he wants to be challenged. $\mathscr{C}$ chooses $\lambda \in\{0,1\}$ at random and implements the following process.

(i) $\mathscr{C}$ sets $S^{*} \longleftarrow b \cdot P$.

(ii) Let $U_{i}=\left(k_{I D_{i}} x+t_{i}\right) S^{*}+r_{i} X$, and compute $\mu_{i}=$ $H_{3}\left(U_{i}, I D_{i}, p k_{i}\right), i=1,2, \cdots, n$.

(iii) $\mathscr{C}$ chooses $e \in Z_{q}^{*}$ at random and computes a polynomial $f(x)$ with degree $n$ as follows:

$$
\begin{aligned}
f(x) & =\prod_{i=1}^{n}\left(x-\mu_{i}\right)+e(\bmod q) \\
& =x^{n}+a_{n-1}^{*} x^{n-1}+\cdots+a_{1}^{*} x+a_{0}^{*}, \\
& \quad \text { where } a_{i} \in Z_{q}^{*}(i=0,1, \cdots, n-1)
\end{aligned}
$$

(iv) $\mathscr{C}$ chooses $\tau^{*} \in\{0,1\}^{l_{1}+1_{2}}$ and $\omega^{*} \in\{0,1\}^{l_{2}}$ at random and computes $C^{*}=\tau^{*} \oplus\left(m_{\lambda} \| \omega^{*}\right)$. 
Final, $\mathscr{C}$ sends the ciphertext $\sigma^{*}=\left(S^{*}, C^{*}, f^{*}\right)$.

Phase 3. In this phase, $\mathscr{A}_{1}$ can make the same queries in Phase 2 except that he cannot make decryption queries with $I D^{*} \in\left\{I D_{\lambda}, I D_{2}, \cdots, I D_{n}\right\}$ and $\sigma^{*}$.

In this phase, $\mathscr{A}_{1}$ can make the same queries in Phase 2 except that he cannot make decryption queries with $I D^{*} \epsilon$ $\left\{I D_{\lambda}, I D_{2}, \cdots, I D_{n}\right\}$ and $\sigma^{*}$.

Guess. $\mathscr{A}_{1}$ outputs $\lambda^{\prime} \in\{0,1\}$ as his/her guess value about $\lambda$. If $\lambda=\lambda^{\prime}$, then $\mathscr{C}$ outputs 1 ; otherwise, $\mathscr{C}$ outputs $0 . \mathscr{A}_{1}$ wins the game if and only if $\lambda=\lambda^{\prime}$ holds.

$\mathscr{A}_{1}$ outputs $\lambda^{\prime} \in\{0,1\}$ as his/her guess value about $\lambda$. If $\lambda=\lambda^{\prime}$, then $\mathscr{C}$ outputs 1 ; otherwise, $\mathscr{C}$ outputs $0 . \mathscr{A}_{1}$ wins the game if and only if $\lambda=\lambda^{\prime}$ holds.

Based on the above oracle queries, the simulation of $\mathscr{C}$ is perfect. Next, we consider the probability that challenger $\mathscr{C}$ fails in Game I. Combined with the previous description, we know that $\mathscr{C}$ fails in Decryption query if $\left(S_{i}, e_{i}\right)$ is not in $L_{H_{4}}^{\text {list }}$. The probability that $\mathscr{A}_{1}$ can correctly guess the output of $H_{4}:\{0,1\}^{*} \longrightarrow\{0,1\}^{l_{1}+l_{2}}$ is $1 / 2^{l_{1}+l_{2}}$. Therefore, the probability of $\mathscr{C}$ failure in Game I is less than $q_{d} / 2^{l_{1}+l_{2}}$, where $q_{d}$ denotes the decryption query times in the game.

If $X=a b P$ holds, then $\sigma^{*}$ is valid ciphertext. Thus, $\mathscr{A}_{1}$ is able to distinguish $\lambda$ with nonnegligible advantage $\epsilon$.

$$
\begin{aligned}
\operatorname{Pr}[c=1 \mid X=a b P] & =\operatorname{Pr}\left[\lambda=\lambda^{\prime} \mid X=a b P\right] \\
& =\frac{1}{2}+\epsilon .
\end{aligned}
$$

If $X \neq a b P$, then the ciphertext distribution is random and uniform when $\lambda=0$ or $\lambda=1$, so $\mathscr{A}_{1}$ cannot distinguish $\lambda$ with any advantage.

$$
\operatorname{Pr}[c=1 \mid X \neq a b P]=\operatorname{Pr}\left[\lambda=\lambda^{\prime} \mid X \neq a b P\right]=\frac{1}{2} .
$$

Therefore, if $\mathscr{A}_{1}$ can break the ANON-IND-sMID-CCA security of the proposed CLAMRE scheme with nonnegligible advantage $\epsilon$, then challenger $\mathscr{C}$ can solve the DDH problem with a nonnegligible advantage $\epsilon-q_{d} / 2^{l_{1}+l_{2}}$. Because the DDH problem is difficult, the proposed CLAMRE scheme is ANON-IND-sMID-CCA secure against $\mathscr{A}_{1}$.

Theorem 7. In the random oracle model, our proposed CLAMRE scheme is ANON-IND-sID-CCA secure against the adversary $\mathscr{A}_{2}$ with the hardness assumption of $C D H$ problem.

Proof. The proof of this theorem is similar to that of Theorem 5 . To save space, we will not give the details here.

\section{Performance Analysis}

In this section, we mainly analyzed computational cost of the proposed CLAMRE scheme. The proposed CLAMRE scheme is compared with Hung et al.s CLAMRE scheme (Hung et al. 2015) and He et al.s CLAMRE scheme (He et al. 2017) to calculate cost.
TABLE 1: Runtime of related operations.

\begin{tabular}{lc}
\hline Notations & Runtime (milliseconds) \\
\hline$T_{b p}$ & 32.713 \\
$T_{h t p}$ & 33.582 \\
$T_{s m-G_{1}}$ & 13.405 \\
$T_{a d d-G_{1}}$ & 0.056 \\
$T_{\text {exp-G }}$ & 2.249 \\
$T_{m u l-G_{2}}$ & 0.008 \\
$T_{s m-\widehat{G}}$ & 3.335 \\
$T_{a d d-\widehat{G}}$ & 0.014 \\
$T_{g h}$ & 0.006 \\
$T_{s c}$ & 0.001 \\
\hline
\end{tabular}

Let $G_{1}$ be an additive group defined on a super singular elliptic curve over a prime field $F_{p}$ with the prime order $q$, and the lengths of $q$ and $p$ are 512 bits and 160 bits, respectively. The Tate bilinear pairing $\hat{e}: G_{1} \times G_{1} \longrightarrow G_{2}$, in order to achieve the same security. For the CLAME scheme based on the elliptic curve cryptography, we also think about an additive group $\widehat{G}$ defined on a nonsingular elliptic curve over a prime field $F_{p}$ with the prime order $q$; lengths of $p$ and $q$ are 160 bits. For convenience, the concept of runtime for some cryptographic operations is defined as follows.

(i) $T_{b p}$ is the runtime required for computing a bilinear pairing.

(ii) $T_{H T P}$ is the runtime required for finishing a hash-topoint operation.

(iii) $T_{s m-G_{1}}$ is the runtime required for computing a scale multiplication in $G_{1}$.

(iv) $T_{a d d-G_{1}}$ is the runtime required for computing an addition in $G_{1}$.

(v) $T_{m u l-G_{2}}$ is the runtime required for computing a multiplication in $G_{2}$.

(vi) $T e_{x p-G_{2}}$ is the runtime required for executing an exponentiation operation in $G_{2}$.

(vii) $T_{s m-\widehat{G}}$ is the runtime required for computing a scale multiplication in $\widehat{G}$.

(viii) $T_{a d d-\widehat{G}}$ is the runtime required for computing an addition in $\widehat{G}$.

(ix) $T_{g h}$ is the runtime required for executing a general hash operation.

(x) $T_{s c}$ is the runtime required for executing a symmetric cryptography operation.

He et al.[30] have implemented related operations on a mobile phone (Samsung Galaxy S5 with a Quad-core $2.45 \mathrm{G}$ processor, $2 \mathrm{G}$ bytes memory, and the Google Android 4.4.2 operating system) using a famous cryptographic library (MIRACL). The implementation results are shown in Table 1.

We denote $n$ the number of the receivers. In order to encrypt a given message $m$, in Hung et al.'s CLAMRE scheme, the sender needs to perform $2 n$ scale multiplication operations in $G_{1}, n$ bilinear pairing operations, $n$ exponentiation operations in $G_{2}, n$ hash-to-point operations, $3 n+2$ general hash operations, and one symmetric cryptography operation. Therefore, in Hung et al.s CLAMRE scheme, the runtime 
TABLE 2: Comparison of runtime (milliseconds).

\begin{tabular}{lcc}
\hline & Encryption & Decryption \\
\hline Hung's scheme & $95.372 \times n+0.013$ & 46.149 \\
He' scheme & $10.044 \times n+3.348$ & 6.714 \\
Our scheme & $6.704 \times n+3.348$ & 6.689 \\
\hline
\end{tabular}

of the sender is $n \times T_{b p}+n \times T_{h t p}+2 n \times T_{s m-G_{1}}+n \times$ $T_{\text {exp-G }}+(3 n+2) \times T_{g h}+T_{s c}=n \times 32.713+n \times 33.582+$ $2 n \times 13.405+n \times 2.249+(3 n+2) \times 0.006+0.001=$ $(95.372 \times n+0.013) \mathrm{ms}$. For decrypting the received ciphertext, receiver needs to implement following operations: one scale multiplication in $G_{1}$, one bilinear pairing, five general hash, and one symmetric cryptography operations. Therefore, the runtime of the receiver in Hung et al's CLAMRE scheme is $T_{b p}+T_{s m-G_{1}}+5 \times T_{g h}+T_{s c}=32.713+13.405+5 \times 0.006+$ $0.001=46.149 \mathrm{~ms}$.

In He et al.'s [29] scheme, to encrypt a given message $m$, the sender needs to perform the following operations: $n$ times addition in $\widehat{G}, 3 n+1$ times scale multiplication in $\widehat{G}$, $4 n+2$ times general hash, and one symmetric encryption operation and $n$ times exclusive or operation (here, exclusive or operation is approximately equal to symmetric encryption operation). Therefore, the runtime of encryption is $(3 n+$ 1) $\times T_{s m-\widehat{G}}+n \times T_{a d d-\widehat{G}}+(4 n+2) \times T_{g h}+(n+1) T_{s c}=$ $(3 n+1) \times 3.335+n \times 0.014+(4 n+2) \times 0.006+(n+$ 1) $0.001=(10.044 n+3.348) \mathrm{ms}$. In order to get plaintext from the received ciphertext, the receiver needs to finish seven general hash operations, two scale multiplication operations in $\widehat{G}$, one symmetric encryption operation, and one exclusive or operation. Therefore, the runtime of the receiver in our scheme is $2 \times T_{s m-\widehat{G}}+7 \times T_{g h}+2 T_{s c}=6.714 \mathrm{~ms}$.

In the proposed CLAMRE scheme, to encrypt a given message $m$, the sender needs to perform the following operations: $2 n$ times addition in $\widehat{G}, 2 n+1$ times scale multiplication in $\widehat{G}, n+2$ times general hash, and one exclusive or operation. Therefore, in our CLAMRE scheme, the runtime of the sender is $(2 n+1) \times T_{s m-\widehat{G}}+2 n \times T_{a d d-\widehat{G}}+(n+2) \times T_{g h}+$ $T_{s c}=(2 n+1) \times 3.335+2 n \times 0.014+(n+2) \times 0.006+$ $0.001=(6.704 n+3.348) \mathrm{ms}$. In order to get plaintext from the received ciphertext, the receiver needs to finish three general hash operations, two scale multiplication operations in $\widehat{G}$, and one exclusive or operation. Therefore, the runtime of the receiver in our scheme is $2 \times T_{s m-\widehat{G}}+3 \times T_{g h}+T_{s c}=6.689 \mathrm{~ms}$.

We list the runtime of encryption and decryption in Huang et al's scheme, He et al.s scheme, and our scheme in Table 2. For a more intuitive understanding, we also present the runtime of multiencryption algorithms in Figure 2. According to comparisons in Table 2 and Figure 2, we can conclude that the proposed CLAMRE scheme has much less runtime in both encryption and decryption than the recent scheme. Therefore, our proposed CLAMRE scheme has better performance.

\section{Conclusion}

In order to keep up with the rapid development of mobile Internet, in this study, we proposed an efficient CLAMRE

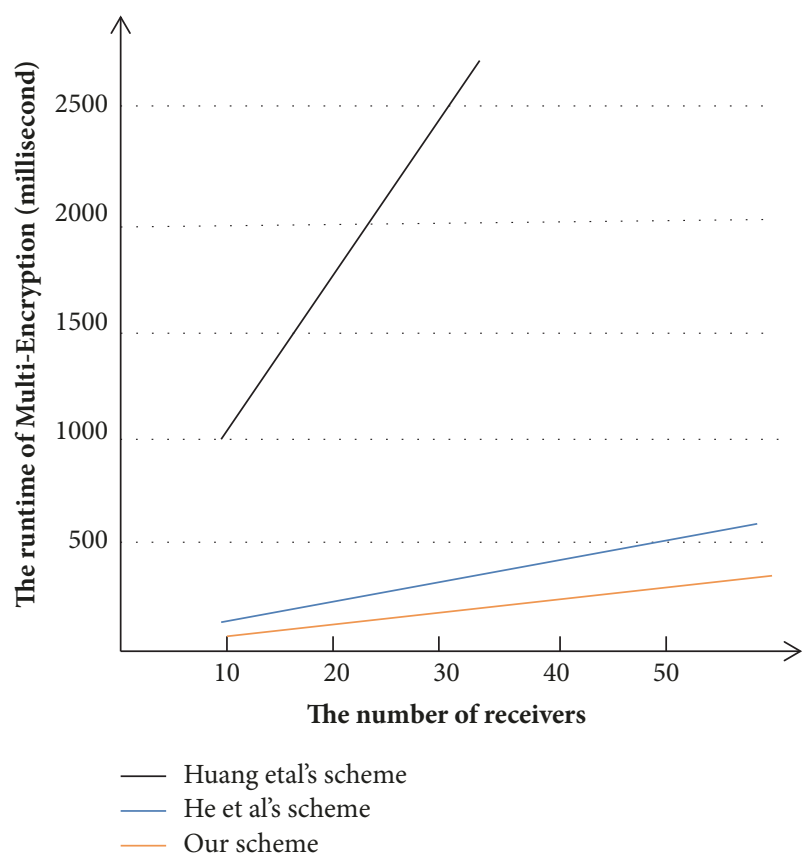

FIGURE 2: Runtime comparison of multiencryption (milliseconds).

scheme using the elliptic curve cryptography. By comparing with recent literature, it shows that our scheme has better performance. We also demonstrate that the proposed CLAMRE scheme provides message confidentiality and protects the privacy of receiver under the random oracle model with the difficulties of decision Diffie-Hellman problem and against the adversaries defined in CL-PKC system.

In summary, our CL-MRE scheme has the following merits: (1) in encryption and decryption process, not using bilinear pairing and probabilistic HTP hash function; (2) achieving confidentiality of message and protecting the privacy of receiver; (3) resisting all known security attacks; (4) low computation and communication costs; (5) avoidance of private key escrow problem and public key certificate management; (6) provable security against IND-sMID-CAA and ANON-IND-sID-CAA under the random oracle.

\section{Data Availability}

The data used in our manuscript was the runtime of some cryptographic operations. He et al. have implemented the runtime of the relevant operations on a mobile phone (Samsung Galaxy S5 with a Quad-core2.45G processor, 2G bytes memory, and the Google Android 4.4.2 operating system) using a famous cryptographic library (MIRACL) in literature [30]. The data (the runtime of some cryptographic operations) used to support the findings of this study is derived from literature $[29,30]$.

\section{Conflicts of Interest}

The authors declare that there are no conflicts of interest regarding the publication of this paper. 


\section{Acknowledgments}

This research is supported by the National Natural Science Foundation of China under Grant no. 61562012; the Innovation Group Major Research Projects of Department of Education of Guizhou Province under Grant no. KY[2016]026.

\section{References}

[1] Z. Fu, K. Ren, J. Shu, X. Sun, and F. Huang, "Enabling personalized search over encrypted outsourced data with efficiency improvement," IEEE Transactions on Parallel and Distributed Systems, vol. 27, no. 9, pp. 2546-2559, 2016.

[2] Z. Fu, X. Sun, Q. Liu, L. Zhou, and J. Shu, "Achieving efficient cloud search services: multi-keyword ranked search over encrypted cloud data supporting parallel computing," IEICE Transactions on Communications, vol. E98B, no. 1, pp. 190-200, 2015.

[3] Z. Xia, X. Wang, X. Sun, Q. Liu, and Q. Wang, "A secure and dynamic multi-keyword ranked search scheme over encrypted cloud data," IEEE Transactions on Parallel and Distributed Systems, vol. 27, no. 2, pp. 340-352, 2016.

[4] X. Huang, Y. Xiang, E. Bertino, J. Zhou, and L. Xu, "Robust multi-factor authentication for fragile communications," IEEE Transactions on Dependable and Secure Computing, vol. 11, no. 6, pp. 568-581, 2014.

[5] P. Guo, J. Wang, B. Li, and S. Lee, "A variable threshold-value authentication architecture for wireless mesh networks," Journal of Internet Technology, vol. 15, no. 6, pp. 929-935, 2014.

[6] J. Shen, H. Tan, J. Wang, J. Wang, and S. Lee, "A novel routing protocol providing good transmission reliability in underwater sensor networks," Journal of Internet Technology, vol. 16, no. 1, pp. 171-178, 2015.

[7] X. Huang, Y. Xiang, A. Chonka, J. Zhou, and R. H. Deng, "A generic framework for three-factor authentication: Preserving security and privacy in distributed systems," IEEE Transactions on Parallel and Distributed Systems, vol. 22, no. 8, pp. 1390-1397, 2011.

[8] Y. Ren, J. Shen, J. Wang, J. Han, and S. Lee, "Mutual verifiable provable data auditing in public cloud storage," Journal of Internet Technology, vol. 16, no. 2, pp. 317-323, 2015.

[9] J. Wang, X. Chen, X. Huang, I. You, and Y. Xiang, "Verifiable auditing for outsourced database in cloud computing," Institute of Electrical and Electronics Engineers. Transactions on Computers, vol. 64, no. 11, pp. 3293-3303, 2015.

[10] C.-C. Lee, Y.-M. Lai, C.-L. Chen, and L. A. Chen, "A novel designated verifier signature scheme based on bilinear pairing," Information Technology and Control, vol. 42, no. 3, pp. 247-252, 2013.

[11] K. Kurosawa, "Multi-recipient Public-Key Encryption with Shortened Ciphertext," in Public Key Cryptography, vol. 2274 of Lecture Notes in Computer Science, pp. 48-63, Springer, Berlin, Germany, 2002.

[12] M. Bellare, A. Boldyreva, and S. Micali, "Public-key encryption in a multi-user setting: security proofs and improvements," in Advances in Cryptology-(EUROCRYPT '2000), B. Preneel, Ed., vol. 1807 of Lecture Notes in Computer Science, pp. 259-274, Springer, Berlin, Germany, 2000.

[13] Y. Dodis and N. Fazio, "Public key broadcast encryption for stateless receivers," in Security and Privacy in Digital Rights
Management, ACM CCS-9 Workshop, J. Feigenbaum, Ed., vol. 2696 of Lecture Notes in Computer Science, pp. 61-80, Springer, Berlin, Germany, 2003.

[14] K. Kurosawa, "Multi-recipient Public-Key Encryption with Shortened Ciphertext," in Public Key Cryptography, vol. 2274 of Lecture Notes in Computer Science, pp. 48-63, Springer Berlin Heidelberg, Berlin, Germany, 2002.

[15] M. Bellare, A. Boldyreva, and D. Pointcheval, "Multirecipient encryption schemes: security notions and randomness re-use," in Proceedings of the Advances in Cryptology (PKC 03, LNCS 2567, pp. 85-99, Miami, Florida, USA, 2003.

[16] J. Baek, R. Safavi-Naini, and W. Susilo, "Efficient multi-receiver identity-based encryption and its application to broadcast encryption," in Public key cryptography-PKC 2005, vol. 3386 of Lecture Notes in Computer Science, pp. 380-397, Springer, Berlin, Germany, 2005.

[17] S. Chatterjee and P. Sarkar, "Multi-receiver identity-based key encapsulation with shortened ciphertext," in Progress in cryptology-INDOCRYPT 2006, vol. 4329 of Lecture Notes in Computer Science, pp. 394-408, Springer, Berlin, Germany, 2006.

[18] J. H. Park and D. H. Lee, "Security analysis of a multireceiver identity-based key encapsulation mechanism," IEICE Transactions on Fundamentals of Electronics, Communications and Computer Sciences, vol. E92-A, no. 1, pp. 329-331, 2009.

[19] H. Wang, P. Zeng, and K.-K. R. Choo, "MDMR-IBE: Efficient multiple domain multi-receiver identity-based encryption," Security and Communication Networks, vol. 7, no. 11, pp. 16411651, 2014.

[20] C. Fan I, L. Huang Y, and H. Ho P, "Anonymous multireceiver identity-based encryption," IEEE Transactions on Computers, vol. 59, no. 9, pp. 1239-1249, 2010.

[21] H.-Y. Chien, "Improved anonymous multi-receiver identitybased encryption," The Computer Journal, vol. 55, no. 4, pp. 439446, 2012.

[22] H. Wang, "Insecurity of improved anonymous multi-receiver identity-based encryption," The Computer Journal, vol. 57, no. 4, pp. 636-638, 2014.

[23] J. Zhang and J. Mao, "An improved anonymous multi-receiver identity-based encryption scheme," International Journal of Communication Systems, vol. 28, no. 4, pp. 645-658, 2015.

[24] S. S. Al-Riyami and K. G. Paterson, "Certificateless public key cryptography," in Advances in Cryptology-ASIACRYPT, vol. 2894 of Lecture Notes in Computer Science, pp. 452-473, Springer, 2003.

[25] Y. Chen, W. Xu, and H. Xiong, "Strongly secure certificateless key-insulated signature secure in the standard model," Annals of Telecommunications-Annales des Télécommunications, vol. 70, no. 9-10, pp. 395-405, 2015.

[26] H. Du and Q. Wen, "Certificateless proxy multi-signature," Information Sciences, vol. 276, pp. 21-30, 2014.

[27] S. H. Islam, M. K. Khan, and A. M. Al-Khouri, "Anonymous and provably secure certificateless multireceiver encryption without bilinear pairing," Security and Communication Networks, vol. 8, no. 13, pp. 2214-2231, 2015.

[28] Y. Hung, S. Huang, Y. Tseng, and T. Tsai, "Efficient Anonymous Multireceiver Certificateless Encryption," IEEE Systems Journal, vol. 11, no. 4, pp. 2602-2613, 2017.

[29] D. He, H. Wang, L. Wang, J. Shen, and X. Yang, "Efficient certificateless anonymous multi-receiver encryption scheme for 
mobile devices," Soft Computing, vol. 21, no. 22, pp. 6801-6810, 2017.

[30] D. He, S. Zeadally, N. Kumar, and W. Wu, "Efficient and Anonymous Mobile User Authentication Protocol Using Self-Certified Public Key Cryptography for Multi-Server Architectures," IEEE Transactions on Information Forensics and Security, vol. 11, no. 9, pp. 2052-2064, 2016. 


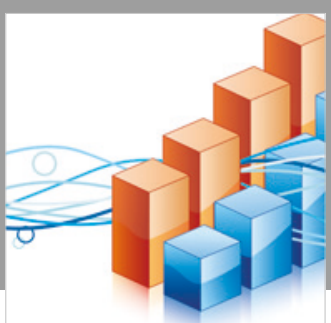

Advances in

Operations Research

\section{-n-m}
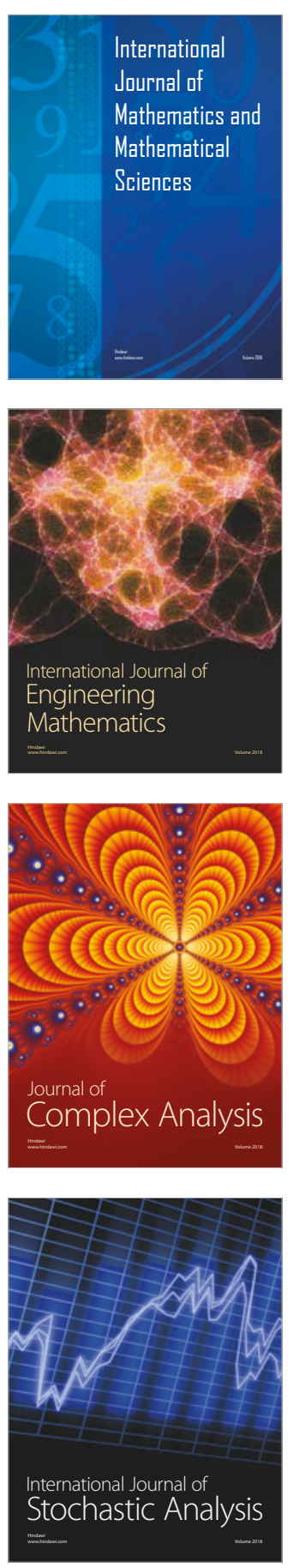
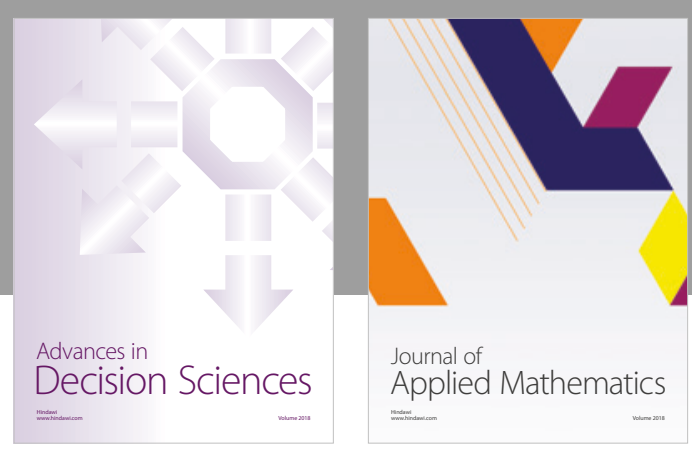

Journal of

Applied Mathematics
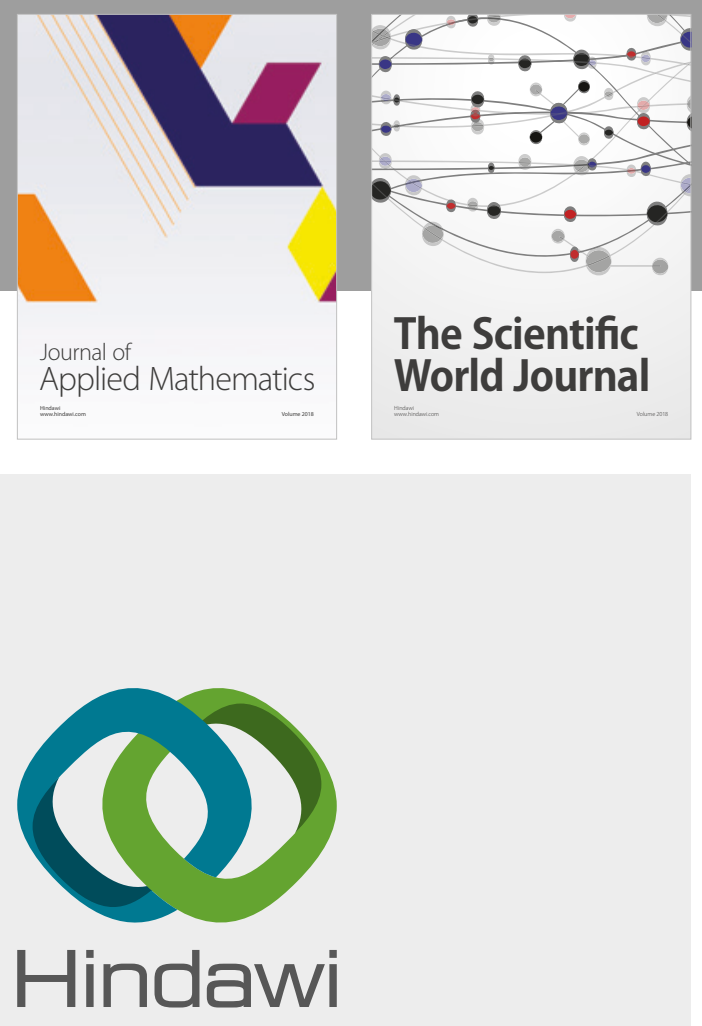

Submit your manuscripts at

www.hindawi.com

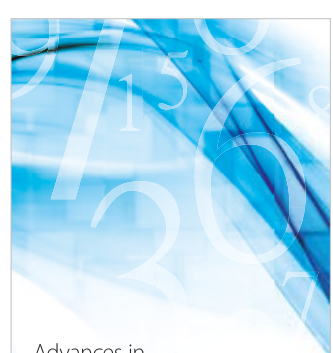

Advances in
Numerical Analysis
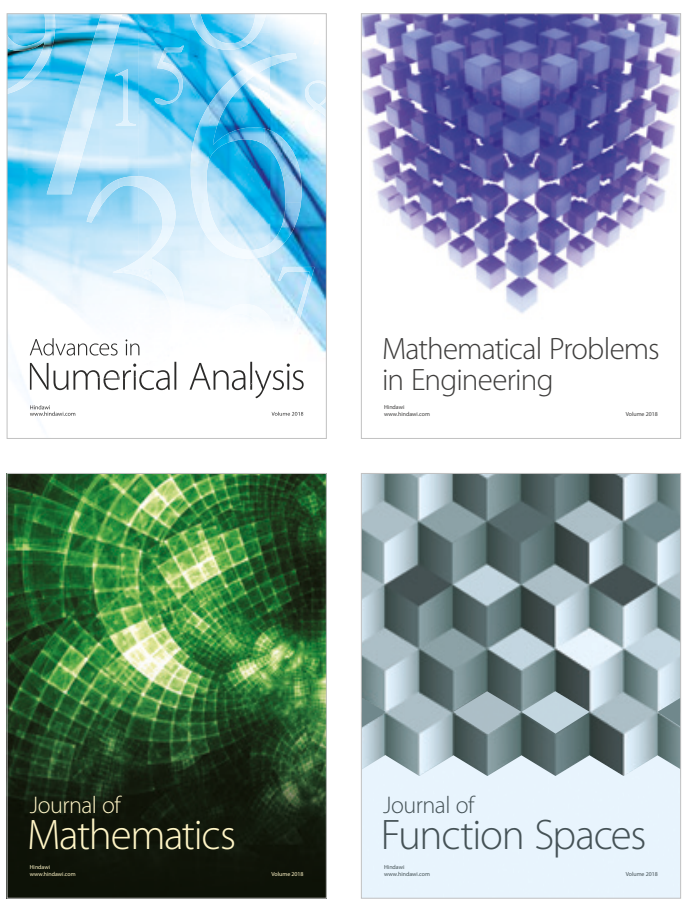

Mathematical Problems in Engineering

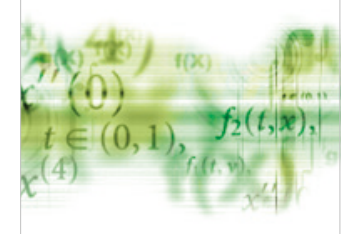

International Journal of

Differential Equations

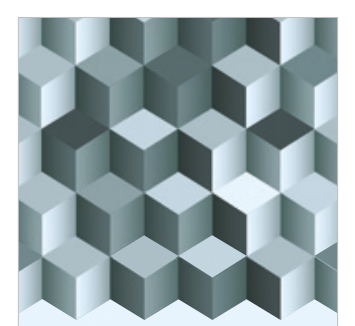

Journal of

Function Spaces

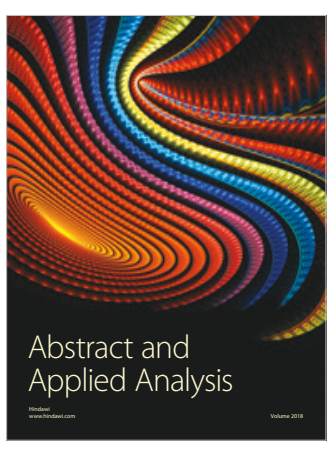

The Scientific

World Journal

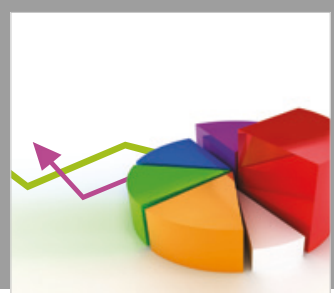

Journal of

Probability and Statistics
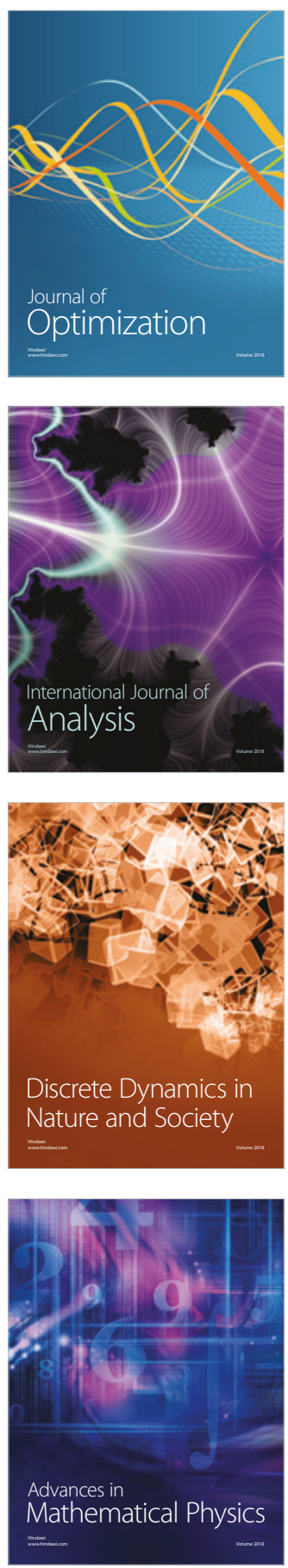